\title{
On a stochastic model of a cascade
}

by

\section{K. URBANIK (Wroctaw)}

1. The homogeneous regular Markov process with a denumerable multitude of states is defined as a stochastic process $\left\langle\Omega, 73_{\Omega}, P r\right\rangle$ satisfying the following conditions:

$1^{\circ}$ the space $\Omega$ of the process consists of integral-valued step functions $\omega(i$. e., functions having only a finite set of points of discontinuity in every finite interval) taking on non-negative values, defined for $t \geqslant 0$, continuous on the right and assuming the same value at $t=0: \omega(0)=n_{0}$;

$2^{\circ} C_{\Omega}$ is the smallest $\sigma$-field spanned upon the sets of form

$$
A(t, n)=\{\omega: \omega(t)=n\}
$$

$3^{\circ}$ there exists a family of functions $P_{n}^{k}(t)(n, k=0,1, \ldots ; t \geqslant 0)$ called the transition probabilities from the state $n$ to the state $k$ in the time interval $t$ satisfying the conditions:

$$
\begin{aligned}
\sum_{k=0}^{\infty} P_{n}^{k}(t) & =1, \quad P_{n}^{k}(t) \geqslant 0, \\
P_{n}^{k}\left(t_{1}+t_{2}\right) & =\sum_{s=0}^{\infty} P_{s}^{k}\left(t_{1}\right) P_{n}^{s}\left(t_{2}\right), \\
\lim _{t \rightarrow 0 \dagger} P_{n}^{k}(t) & =P_{n}^{k}(0)=\delta_{n}^{k},
\end{aligned}
$$

(5) if $t_{1}<t_{2}<\ldots<t_{r}$, then $\operatorname{Pr}\left(\bigcap_{i=1}^{r}\left\{\omega: \omega\left(t_{i}\right)=n_{i}\right\}\right)=\prod_{i=1}^{r} P_{n_{i-1}}^{n_{i}}\left(t_{i}-t_{i-1}\right)$, where $t_{0}=0$ (see, for instance, [3], p. 236).

It follows from theorems of Doob and Kolmogorov ([2], p. 468, [7], p. 53) that for the transition probabilities in the homogeneous regular Markor processes there exist limits

$$
\begin{gathered}
a_{n !}^{k}=\lim _{t \rightarrow 0+\downarrow} \frac{P_{n}^{k}(t)}{t} \quad \text { for } \quad k \neq n, \\
a_{n}^{n}=\lim _{t \rightarrow 0+} \frac{1-P_{n}^{n}(t)}{t},
\end{gathered}
$$


called the intensities of the process. The intensities of the process satisfy the following conditions:

$$
\begin{gathered}
a_{n}^{k} \geqslant 0 \quad(k=0,1, \ldots), \\
a_{n}^{n}=\sum_{k \neq n} a_{n}^{k} .
\end{gathered}
$$

When the intensities of the process are known, the transition probabilities of the process may be determined from the Kolmogorov equations (compare [4], p. 64)

$$
\frac{d}{d t} P_{n}^{k}(t)=-a_{k}^{k} P_{n}^{k}(t)+\sum_{s \neq k} a_{s}^{k} P_{n}^{s}(t)
$$

with the initial values

$$
P_{n}^{k}(0)=\delta_{n}^{k}
$$

Thus it follows from condition (5) that the intensities determine the probability $P r$ in the process satisfying conditions $1^{\circ}$ and $2^{\circ}$. It follows that if the intensities of two homogeneous regular Markov processes are the same and the realizations of both the processes assume the same value at zero, then the processes are identical.

The function $\left.P(A / B)(A, B \in\urcorner \beta_{\Omega}\right)$ is called the conditional probability if it satisfies the equation

$$
\operatorname{Pr}(A \cap B)=P(A / B) \operatorname{Pr}(B),
$$

and if, for fixed $B$, it is a normed $\sigma$-measure.

2. Let us consider a physical cascade composed of particles of the same kind in a homogeneous medium (e.g., the cascade of nucleons in cosmic radiation) in which the decomposition of the particles is a random event. For physical reasons we assume that

(i) the future of a particle does not depend on its past and depends only on its actual state,

(ii) the destiny of a particle and its progeny does not depend on the future of the actually existing particles.

It is customary to treat mathematically a cascade arising from a single particle as a homogeneous regular Markov process with a denumerable multitude of states. In this process the value of the function $\omega$ for the argument $t$ is equal to the number of the particles in the cascade at the moment $t$; hence for all the realizations we have

$$
\omega(0)=1 \text {. }
$$

One assumes as an intuitive consequerce of (i) and (ii) that the transition probabilities $P_{n}^{k}(t)$ satisfy the supplementary condition

$$
\begin{aligned}
& P_{0}^{k}(t)=\delta_{0}^{k} \quad(k=0,1, \ldots), \\
& P_{n}^{k}(t)=\sum \prod_{i=1}^{n} P_{1}^{k_{i}(t)} \quad(k=0,1, \ldots ; n=1,2, \ldots),
\end{aligned}
$$

the summation being extended over all the systems of non-negative integers $k_{1}+k_{2}+\ldots+k_{n}=k$ (see, for instance, [1], p. 7; [5], p. 409). In terms of intensities condition (14) becomes

$$
a_{n}^{k}=n a_{1}^{k-n+1} \quad(n, k=0,1, \ldots) .
$$

Then the Kolmogorov equations (10) change into

$$
\frac{d}{d t} P_{n}^{k}(t)=-k a_{1}^{1} P_{n}^{k}(t)+\sum_{s=1}^{k-1} s a_{1}^{k-s+1} P_{n}^{s}(t)+(k+1) a_{1}^{0} P_{n}^{k+1}(t) .
$$

In this model of the cascade we cannot, of course, deal with individual particles and their future, whence conditions (i) and (ii) have no direct interpretation. Thus in the precise treatment of the cascade processes conditions (14) or (15) are considered as axioms.

Professor E. Marczewski has proposed a construction of a mathematical model of a cascade in which it would be possible to deal with the individual particles and their future, and which would be based upon the conditions (i) and (ii). We shall now be concerned with the investigation of such a model.

3. Let $\Xi$ be the set of all the systems

$$
\xi=\left\langle x_{1}, x_{2}, \ldots, x_{n}\right\rangle \quad(n=1,2, \ldots),
$$

where $x_{1}=1, x_{k}=\langle i, j\rangle(k=2,3, \ldots ; i=1,2, \ldots, j ; j=2,3, \ldots)$.

Let $V$ be the space of all the functions $v$ defined on $\Xi$, whose values are intervals of the form $[a, b)$ lying on the positive semi-axes (i.e., $0 \leqslant a \leqslant b \leqslant \infty)$ and satisfying the conditions

(a) $v(\langle 1\rangle)=[0, a) \quad(0<a \leqslant \infty)$;

(b) if $v\left(\left\langle x_{1}, \ldots, x_{n}\right\rangle\right)=[a, b)$, then $v\left(\left\langle x_{1}, \ldots, x_{n}, x_{n+}\right\rangle\right)=[b, c)$;

(c) if $\left.v\left(\left\langle x_{1}, \ldots, x_{n},\langle i, j\rangle\right\rangle\right) \neq 0^{1}\right)$, then $v\left(\left\langle x_{1}, \ldots, x_{n},\langle k, j\rangle\right\rangle\right) \neq 0$ for $k=1,2, \ldots, j$;

$v\left(\left\langle x_{1}, \ldots, x_{n},\langle s, m\rangle\right\rangle\right)=0$ for $s=1,2, \ldots, m ; m \neq j$;

(d) if $v\left(\left\langle x_{1}, \ldots, x_{n}\right\rangle\right)=0$, then $v\left(\left\langle x_{1}, \ldots, x_{n}, x_{n+1}\right\rangle\right)=0$;

(e) if $v\left(\left\langle x_{1}, \ldots, x_{n}\right\rangle\right) \neq 0$ for $n=1,2, \ldots$, then

$$
\bigcup_{n=1}^{\infty} v\left(\left\langle x_{1}, \ldots, x_{n}\right\rangle\right)=[0, \infty)
$$

1) 0 denotes here the empty interval $[a, a)$. 
The intuitive sense of the elements $\xi$ and the function $v$ is the following one:

The element $\xi=\langle 1\rangle$ represents the particle generating the cascade; the element $\xi=\langle 1,\langle i, j\rangle\rangle$ represents the $i$-th particle arising by the decomposition into $j$ parts of the particle generating the cascade; generally, the element $\xi=\left\langle x_{1}, \ldots, x_{n},\langle i, j\rangle\right\rangle$ represents the $i$-th particle generated by the decomposition of the particle represented by the element $\left\langle x_{1}, \ldots, x_{n}\right\rangle$ into $j$ new particles; $v(\xi)=[a, b)$ where $a<b$ means that the particle represented by the system $\xi$ arose at the moment $a$ and finished its life, $i$. e., was decomposed by the medium, at the moment $b$; $v(\xi)=[a, a)=0$ means that a particle represented by $\xi$ does not exist: in the cascade. Hence the function $v$ describes the history of all the particles in a given cascade. Condition (a) states that the cascade arises from a single particle at the moment $t=0,(d)$ states that the non-existing particle cannot generate new particles, finally, (e) states that in every finite time interval only a finite number of decompositions can happen.

The following lemmas will show that condition (e) in the definition of the space $V$ may be replaced by

$\left(\mathrm{e}^{\prime}\right)$ for every $T<\infty$ the set $\{\xi: v(\xi) \cap[0, T) \neq 0\}$ is finite.

LEMca 1. Condition ( $\left.e^{\prime}\right)$ is satisfied for every $v \in \nabla$.

Proof. Arguing indirectly, suppose that there is a $v_{0} \in V$ such that $\left(\mathrm{e}^{\prime}\right)$ is not satisfied. Then there exists a number $T_{0}$ such that the set

$$
\Xi_{0}=\left\{\xi: v_{0}(\xi) \frown\left[0, T_{0}\right) \neq 0\right\}
$$

is infinite. Conditions (b) and (d) lead to the following implication:

(17) if $\left\langle x_{1}, \ldots, x_{n}, x_{n+1}\right\rangle \epsilon \Xi_{0}$, then $\left\langle x_{1}, \ldots, x_{n}\right\rangle \in \Xi_{0}$.

From (c) it follows that there is only a finite multitude of elements $x_{r}$ appearing on the $r$-th place in the systems $\left\langle x_{1}, \ldots, x_{r \ldots 1}, x_{r}, x_{r+1}, \ldots, x_{n}\right\rangle$ of the set $\Xi_{0}$. The infinitude of the set $\Xi_{0}$ and condition (17) imply, for every $n$, the existence of a system $\left\langle x_{1}, \ldots, x_{n}\right\rangle$ belonging to $\Xi_{0}$. It follows that there exists a sequence $x_{1}^{0}, x_{2}^{0}, \ldots$ such that $\left\langle x_{1}^{0}, x_{2}^{0}, \ldots, x_{n}^{0}\right\rangle \in \Xi_{0}$ for every $n$ ([8], p. 115): Let us write in accordance with condition (b)

$$
v_{0}\left(\left\langle x_{1}^{0}, x_{2}^{0}, \ldots, x_{n}^{0}\right\rangle\right)=\left[a_{n}, a_{n+1}\right), \quad\left(w_{1}=1,2, \ldots\right) .
$$

From the definition of the set $\Xi_{0}$ and of the sequence $x_{1}^{0}, x_{2}^{0}, \ldots$ we deduce the inequality $a_{n}<T_{0}(n=1,2, \ldots)$, whence from (18) it follows that

$$
\bigcup_{n=1}^{\infty} v_{0}\left(\left\langle x_{1}^{0}, \ldots, x_{n}^{0}\right\rangle\right) \subset\left[0, T_{0}\right)
$$

which, in virtue of the finiteness of $T_{0}$, contradicts condition (e). Thus the lemma is proved.

LEMma 2. Let the function $v$ satisfy conditions (a), $\left(\mathrm{e}^{\prime}\right)$ and $(\mathrm{b})$; then condition (e) is also satisfied.

Proof. Let

$$
v\left(\left\langle x_{1}, \ldots, x_{n}\right\rangle\right) \neq 0 \quad \text { for } \quad n=1,2, \ldots
$$

Conditions (a) and (b) imply

$$
\bigcup_{n=1}^{\infty} v\left(\left\langle x_{1}, \ldots, x_{n}\right\rangle\right)=[0, T)
$$

It follows by (19) that the set $\{\xi: v(\xi) \cap[0, T) \neq 0\}$ is infinite. Hence from $\left(e^{\prime}\right)$ we deduce $T=\infty$, which proves the lemma.

4. Let $\Re(Y, T)$ denote the smallest $\sigma$-field spanned upon the sets of the form

$$
B(\xi, t)=\{v: v \in V, t \in v(\xi)\}
$$

where $\xi \epsilon Y$ and $t \geqslant T$. The set $\{v: v \epsilon V, \Phi(v)\}$ will be denoted shortly by $\{\Phi(v)\}$; thus the set (20) will be denoted by $\{t \epsilon v(\xi)\}$.

We shall now define a family $T_{\tau}$ of transformations of sets belonging to the $\sigma$-field $93(Y, T)$. For the sets of the form $(20)$ we define

$$
T_{\tau}\{t \in v(\xi)\}=\{t-\tau \epsilon v(\xi)\} \quad(\tau \leqslant T),
$$

and then we extend this definition to all the sets of $93(X, T)$ by the formulae

$$
T_{\tau} \bigcup_{i} A_{i}=\bigcup_{i} T_{\tau} A_{i}, \quad T_{\tau} A^{\prime}=\left(T_{\tau} A\right)^{\prime} .
$$

By the definition of $T_{\tau}(\tau \leqslant T)$ it follows for $A \epsilon \beta(Y, T)$ that $T_{\tau} A \epsilon \bigcirc B(Y, T-\tau)$.

Let $\xi=\left\langle x_{1}, \ldots, x_{n}\right\rangle$ and $\eta=\left\langle x_{1}, \ldots, x_{n}, y_{1}, \ldots, y_{m}\right\rangle(m=0,1, \ldots)$; then we shall write $\eta \epsilon K(\xi)$ and $\left.\eta-\xi=\left\langle 1, y_{1}, \ldots, y_{m}\right\rangle^{2}\right)$.

$K(\xi)$ is a cascade arising from the particle $\xi$.

We have, in particular, $K(\langle 1\rangle)=\xi$ and $\eta-\langle 1\rangle=\eta$. If $\eta \in K(\xi)$, we put $L_{\xi}\{t \epsilon v(\eta)\}=\{t \epsilon v(\eta-\xi)\}$, and by the formulae

$$
L_{\xi} \bigcup_{i} A_{i}=\bigcup_{i} L_{\xi} A_{i}, \quad L_{\xi} A^{\prime}=\left(L_{\xi} A\right)^{\prime}
$$

we extend the definition of the transformation $L_{\xi}$ to all the sets belonging to the $\sigma$-field $73(K(\xi), T)$. Let us observe that $A \epsilon \subset B(K(\xi), T)$ implies $\left.L_{\xi} A \epsilon^{C}\right) 3(\xi, t)$.

2) We set $\left\langle x_{1}, \ldots, x_{m}, y_{1}, \ldots, y_{m}\right\rangle=\left\langle x_{1}, x_{2}, \ldots, x_{n}\right\rangle$ for $m=0$. studia Mathematioa XVI. 
The $\sigma$-field $98(\Xi, 0)$ will be denoted by $9 \beta_{V}$. Let $P$ be a probability (= normed $\sigma$-measure) on the $\sigma$-field $9_{V}$, inducing the conditional probability (in the sense of section 1) and satisfying for $A \epsilon \subseteq B(K(\xi), t)$ and $B \epsilon_{\epsilon} \subset B(E-K(\xi), 0)$ the following conditions:

$$
\begin{aligned}
& P(A \mid t \epsilon v(\xi))=P\left(L_{\xi} A \mid t \epsilon v(\langle 1\rangle)\right), \\
& P(A \mid\{t \in v(\xi)\} \cap B)=P(A \mid t \in v(\xi)), \\
& P(A \mid t \epsilon v(\xi))=P\left(T_{\tau} A \mid t-\tau \in v(\xi)\right),
\end{aligned}
$$

where $t \geqslant \tau$.

The system $\left\langle V, 1 B_{K}, P\right\rangle$ will be called a cascade process. Conditions (I) and (II) are transcriptions of conditions (i) and (ii), and condition (III) describes the homogeneity in time of the process.

5. Let $\chi(v(\xi), t)$ be the characteristic function (in the senso of the theory of sets) of the interval $v(\xi)$. Let us write for $v \epsilon V$ and $t \geqslant 0$

$$
\omega(v, t)=\sum_{\xi \in \Xi} \chi(v(\xi), t) .
$$

Condition ( $\left.\mathrm{e}^{\prime}\right)$ implies that $\omega(v, t)$ is a step-function of the variable $t$, taking on non-negative integer values. It is easily verified that the number of particles in the cascade described by the function $v$ is equal at the instant $t$ to $\omega(v, t)$.

Formula (21) defines a mapping of the space $V$ onto the space of all the step-functions $\omega$ defined for $t \geqslant 0$, taking on non-negative integer values and equal to 1 at $t=0$. Let us denote this mapping by $\Omega$. Hence

$$
\begin{aligned}
\Omega(v)(t) & =\omega(v, t), \\
\Omega^{-1}\{\omega: \omega(t)=n\}=\left\{\sum_{\xi \in \Xi} \chi(v(\xi), t)\right. & =n\}=\bigcup_{*} \bigcap_{j=1}^{n}\left\{t \in v\left(\xi_{j}\right)\right\} \frown \bigcap_{*}\{t \notin v(\eta)\},
\end{aligned}
$$

the union $\bigcup_{*}$ being extended to all non-ordered systems $\xi_{1}, \xi_{2}, \ldots, \xi_{m}$ $\left(\xi_{i} \epsilon \xi ; \xi_{i} \neq \xi_{j}\right.$ for $\left.i \neq j\right)$ and the intersection $\bigcap_{*}$ being taken over all the elements $\eta \in \Xi$ different from $\xi_{1}, \xi_{2}, \ldots, \xi_{n}$. Wa get

$$
\Omega^{-1}\{\omega: \omega(t)=n\} \in \subset g_{V},
$$

whence, denoting by $\mathcal{B}_{\Omega(V)}$ the smallest $\sigma$-field spanned upon the sets of the form (1), we see that $A \in \bigcap_{\Omega(V)}$ implies $\Omega^{-1} A \in{ }^{-1} B_{V}$. Thus the mapping $\Omega$ defines in $73_{\Omega(V)}$ a normed $\sigma$-measure which will be denoted by $\Omega P$ : $\Omega P(A)=P\left(\Omega^{-1} A\right)$ for $A \epsilon 93_{\Omega(V)}$. Thus the mapping transforms the cascade process $\left\langle V, 77_{V}, P\right\rangle$ into the stochastic process $\left\langle\Omega(V), 73_{\Omega(V)}, \Omega P\right\rangle$.

THEOREM 1. For every cascade process $\left\langle V, \gamma_{\mathrm{V}}, P\right\rangle$ the corresponding stochastic process $\left\langle\Omega(V), \bigcap_{\Omega(V)}, \Omega P\right\rangle$ is a homogeneous regular Markov process with a denumerable multitude of states, satisfying conditions (13) and (14).

THEOREM 2. For every homogeneous regular Markov process with a denumerable multitude of states $\left\langle\Omega, 93_{\Omega}, P r\right\rangle$, satisfying conditions (13) and (14) there exists a cascade process $\left\langle V, \bigcap_{V}, P\right\rangle$ such that $\left\langle\Omega(V), \bigcap_{\Omega(V)}, \Omega P\right\rangle$ is identical with $\left\langle\Omega, 9 \oiint_{\Omega}, P r\right\rangle$.

Theorems 1 and 2 join the models of stochastic cascades described in sections $2-4$. The proofs of both theorems will be given in the sections to follow (they were announced without proof in the note [9]).

6. We need following lemmas.

LEMMIA 3. Let

$$
K\left(\xi_{i}\right) \cap K\left(\xi_{j}\right)=0 \quad(i \neq j ; i, j=1,2, \ldots, n) ;
$$

then for

$$
\begin{gathered}
A_{i} \in \mathcal{7}\left(K\left(\xi_{i}\right), t\right) \quad(i=1,2, \ldots, n), \\
B \in \Re\left(\Xi-\bigcup_{i=1}^{n} K\left(\xi_{i}\right), 0\right)
\end{gathered}
$$

we have

$$
P\left(\bigcap_{i=1}^{n} A_{i} \cap\left\{t \epsilon v_{(}\left(\xi_{i}\right)\right\}_{\cap} B\right)=\left[\prod_{i=1}^{n} P\left(A_{i} \mid t \epsilon v\left(\xi_{i}\right)\right)\right] \cdot P\left(\bigcap_{i=1}^{n}\left\{t \epsilon v\left(\xi_{i}\right)\right\}_{\cap} B\right) .
$$

Proof. We shall prove this by induction with respect to $n$. For $i=1$, formula (25) follows from condition (II). Suppose that (25) is valid for $n=k$, and let us write

$$
B_{0}=\bigcap_{i=1}^{k} A_{i} \cap\left\{t \epsilon v\left(\xi_{i}\right)\right\} \frown B .
$$

Then we have

$$
B_{0} \epsilon 93\left(\xi-K\left(\xi_{k+1}\right), 0\right)
$$

and

$$
P\left(\bigcap_{j=1}^{k+1} A_{i} \cap\left\{t \epsilon v\left(\xi_{i}\right)\right\} \frown B\right)=P\left(A_{k+1} \cap\left\{t \epsilon v\left(\xi_{k+1}\right)\right\} \cap B_{0}\right) .
$$

Hence by (II) we obtain

$$
P\left(\bigcap_{i=1}^{k+1} A_{i} \frown\left\{t \in v\left(\xi_{i}\right)\right\} \frown B\right)=P\left(A_{k+1} \mid t \in v\left(\xi_{k+1}\right)\right) P\left(\left\{t \in v\left(\xi_{k+1}\right)\right\} \frown B_{0}\right) .
$$

Now, setting

$$
B_{1}=\left\{t \in v\left(\xi_{k+1}\right)\right\} \sim B
$$


we get

$$
P\left(\left\{t \in v\left(\xi_{k+1}\right)\right\} \cap B_{0}\right)=P\left(\bigcap_{i=1}^{k} A_{i} \cap\left\{t \in v\left(\xi_{i}\right)\right\} \cap B_{1}\right),
$$

and in virtue of (22), (23), and (24)

$$
B_{1} \in B\left(\Xi-\bigcup_{i=1}^{k} K\left(\xi_{i}\right), 0\right) \text {. }
$$

By the hypothesis of induction we deduce hence

$$
P\left(\left\{t \in v\left(\xi_{k+1}\right)\right\} \cap B_{0}\right)=\left|\prod_{i=1}^{k} P\left(A_{i} \mid t \in v\left(\xi_{i}\right)\right)\right| \cdot P\left(\bigcap_{i=1}^{k}\left\{t \in v\left(\xi_{i}\right)\right\} \cap B_{1}\right) .
$$

Putting this expression into formula (26) and taking into account formula (27) we obtain formula (25) for $n=k+1$, which proves the lemma.

LEMMNA 4. Let

$$
\begin{gathered}
K\left(\xi_{i}\right) \cap K\left(\xi_{j}\right)=0 \quad(i \neq j), \\
t<T
\end{gathered}
$$

then for every $k$ there exists a set

$$
B \in(\theta):\left(\xi-\bigcup_{i=1}^{n} K\left(\xi_{i}\right), 0\right)
$$

satisfying the equality

$$
\bigcap_{i=1}^{n}\left\{T \in v\left(\xi_{i}\right)\right\} \frown\{\omega(v, t)=k\}=\bigcap_{i=1}^{n}\left\{T \epsilon v\left(\xi_{i}\right)\right\} \cap B .
$$

Proof. By hypothesis (29) and property (b) of the function $v$ it follows that $T \in v(\xi), \eta \in K(\xi), \eta \neq \xi$ implies $t \in v(\eta)$. This leads to

(30) $\bigcap_{i=1}^{n}\left\{T \in v\left(\xi_{i}\right)\right\} \cap\{\omega(v, t)=k\}$

$$
=\bigcup_{s=0}^{k} \bigcap_{i=1}^{n}\left\{T \epsilon v\left(\xi_{i}\right)\right\} \cap\left\{\sum_{\eta \bar{\theta}=\bigcup_{i=1}^{n} K\left(\xi_{i}\right)} \chi(v(\eta), t)=k-8\right\} \cap\left\{\sum_{i=1+1}^{n} \chi\left(v\left(\xi_{i}\right), t\right)=8\right\} .
$$

Let $\xi_{i}=\left\langle x_{1}^{i}, \ldots, x_{m_{i}}^{i}\right\rangle$; then by hypothesis (28)

(31) $\left\langle x_{1}^{i}, \ldots, x_{r}^{i}\right\rangle \in \Xi-\bigcup_{j=1}^{n} K\left(\xi_{j}\right) \quad\left(r<m_{i} ; i=1,2, \ldots, n\right)^{\prime}$.

From inequality (29) and property (b) of the function $v$ it follows that

(32) $\left\{T \in v\left(\xi_{i}\right)\right\} \cap\left\{t \in v\left(\xi_{i}\right)\right\}=\left\{T \in v\left(\xi_{i}\right)\right\} \cap \bigcap_{r=1}^{m_{i}-1}\left\{t \in v\left(\left\langle x_{1}^{i}, \ldots, x_{r}^{i}\right\rangle\right)\right\}$.
Setting

$$
E_{i}^{0}=\bigcap_{r=1}^{m_{i}-1}\left\{t \in v\left(\left\langle x_{1}^{i}, \ldots, x_{r}^{i}\right\rangle\right)\right\}, \quad E_{i}^{1}=V-E_{i}^{0},
$$

we get, in virtue of formula (32),

$$
\begin{aligned}
\left\{\sum_{i=1}^{n} \chi\left(v\left(\xi_{i}\right), t\right)=8\right\} \cap \bigcap_{i=1}^{n}\left\{T \in v\left(\xi_{i}\right)\right\} & \\
& =\left.\bigcap_{j=1}^{n}\left\{T \in v\left(\xi_{j}\right)\right\} \cap\right|_{r_{1}+\ldots+r_{\eta=k-s}} \bigcap_{i=1}^{n} E_{i}^{r_{i}} \mid .
\end{aligned}
$$

Formulae (31) and (33) lead directly to

$$
\bigcup_{r_{1}+\ldots+r_{n}=k-s} \bigcap_{i=1}^{n} E_{i}^{r_{i}} \epsilon \gamma_{3}\left(\Xi-\bigcup_{i=1}^{n} K\left(\xi_{i}\right), 0\right) \text {. }
$$

Let us now write

$$
\left.B=\bigcup_{s=0}^{k}\left\{\sum_{\substack{n \in \tilde{\epsilon}_{i=1}^{n} K\left(\xi_{i}\right) \\ i=1}} z(v(\eta), t)=k-s\right\} \cap \bigcup_{r_{1}+\ldots+r_{n}=k-s} \bigcap_{i=1}^{n} E_{i}^{r_{i}}\right] .
$$

By formula (35) $B \epsilon C / B\left(\Xi-\bigcup_{i=1}^{n} K\left(\xi_{i}\right), 0\right)$.

Equalities (30) and (34) imply that the set $B$ satisfies our lemma.

LEMMA 5. If $T>t>t_{1}>\ldots>t_{m}$ then for every $k, n_{1}, \ldots, n_{m}$ and $n \geqslant 1, m \geqslant 0$ we have

$$
P\left(\{\omega(v, T)=k\} \frown\{\omega(v, t)=n\} \frown \bigcap_{j=1}^{m}\left\{\omega\left(v, t_{j}\right)=n_{j}\right\}\right)
$$$$
=\left[\sum_{k_{1}+\ldots+k_{n}=k} \prod_{i=1}^{n} P\left(\omega(v, T-t)=k_{i}\right)\right] \cdot P\left(\{\omega(v, t)=n\} \cap \bigcap_{j=1}^{m}\left\{\omega\left(v, t_{j}\right)=n_{j}\right\}\right)
$$

(we set $\bigcap_{j=1}^{m}\left\{\omega\left(v, t_{j}\right)=n_{j}\right\}=\Omega(V)$, as $\left.m=0\right)$.

Proof. Using the definition of the function $\omega$ and properties (a) and (b) we may easily verify the following equality:

$$
\begin{aligned}
& \text { (36) } \left.\quad\{\omega(v, T)=k\} \cap\{\omega(v, t)=n\} \frown \bigcap_{j=1}^{m}\left\{\omega\left(v, t_{j}\right)=n_{j}\right\}\right) \\
& =\bigcup_{*} \bigcup_{k_{1}+\ldots+k_{22}=k} \bigcap_{i=1}^{n}\left\{\sum_{\eta \in K\left(\xi_{i}\right)} \chi(v(\eta), T)=k_{i}\right\} \frown\left\{t \epsilon v\left(\xi_{i}\right)\right\} \frown \\
& \cap \bigcap_{n \bar{G} \bigcup_{i=1}^{n} K\left(\xi_{i}\right)}\{t \in v(\eta)\} \frown \bigcap_{j=1}^{m}\left\{\omega\left(v, t_{j}\right)=n_{j}\right\},
\end{aligned}
$$


the union $\bigcup_{*}$ being extended over all non-ordered systems $\xi_{1}, \xi_{2}, \ldots, \xi_{n}$ such that

$$
K\left(\xi_{i}\right) \cap K\left(\xi_{j}\right)=0 \quad \text { as } \quad i \neq j .
$$

By Lemma 4 there exist sets $B_{j}\left(\xi_{1}, \ldots, \xi_{n}\right)$ belonging to the $\sigma$-field $73\left(\Xi-\bigcup_{i=1}^{n} K\left(\xi_{i}\right), 0\right)$ such that

(38) $\bigcap_{i=1}^{n}\left\{t \in v\left(\xi_{i}\right)\right\} \cap\left\{\omega\left(v, t_{j}\right)=n_{j}\right\}=\bigcap_{i=1}^{n}\left\{t \in v\left(\xi_{i}\right)\right\} \cap B_{j}\left(\xi_{1}, \ldots, \xi_{n}\right)$.

Let us seti

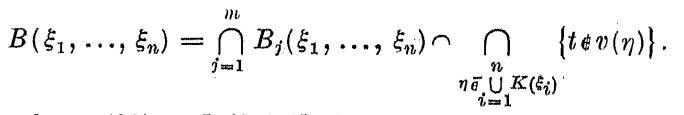

We see from (36) and (38) that

$$
\bigcup_{*} \bigcap_{i=1}^{n}\left\{t \in v\left(\xi_{i}\right)\right\} \cap B\left(\xi_{1}, \ldots, \xi_{n}\right)=\{\omega(v, t)=n\} \cap \bigcap_{j=1}^{m}\left\{\omega\left(v, t_{j}\right)=n_{j}\right\}
$$

$$
B\left(\xi_{1}, \ldots, \xi_{n}\right) \varepsilon \varnothing 8\left(\Xi-\bigcup_{i=1}^{n} K\left(\xi_{i}\right), 0\right) .
$$

Setting

$$
A(\xi, s)=\left\{\sum_{\eta \in \mathcal{K}(\xi)} \chi(v(\eta), T)=s\right\},
$$

and taking into account the fact that the summands on the right side of equality (36) are disjoint, we easily deduce from (36), (38), and (39) that

$$
\text { (43) } \begin{aligned}
P\left(\{\omega(v, T)=k\} \cap\{\omega(v, t)=n\} \cap \bigcap_{j=1}^{n}\left\{\omega\left(v, t_{j}\right)=n_{j}\right\}\right) \\
=\sum_{*} \sum_{k_{1}+\ldots+k_{n}=k} P\left(\bigcap_{i=1}^{n} A\left(\xi_{i}, k_{i}\right) \cap\left\{t \in v\left(\xi_{i}\right)\right\} \cap B\left(\xi_{1}, \ldots, \xi_{n}\right) .\right.
\end{aligned}
$$

By definition (42) we get $A\left(\xi_{i}, k_{i}\right)$ e $\uparrow\left(K\left(\xi_{i}\right), t\right)$. Hence formulae (37) and (4.1) and Lemma 3 lead to

$$
\begin{aligned}
& P\left(\bigcap_{i=1}^{n} A\left(\xi_{i}, k_{i}\right) \cap\left\{t \in v\left(\xi_{i}\right)\right\} \cap B\left(\xi_{1}, \ldots, \xi_{n}\right)\right. \\
& \quad=\left[\prod_{i=1}^{n} P\left(A\left(\xi_{i}, k_{i}\right) \mid t \in v\left(\xi_{i}\right)\right)\right] P\left(\bigcap_{i=1}^{n}\left\{t \in v\left(\xi_{i}\right)\right\} \cap B\left(\xi_{1}, \ldots, \xi_{n}\right)\right) .
\end{aligned}
$$

From conditions (I) and (III) (p. 242) we infer

$$
P\left(A\left(\xi_{i}, k_{i}\right) \mid t \epsilon v\left(\xi_{i}\right)\right)=P\left(T_{t} L_{\xi_{i}} A\left(\xi_{i}, k_{i}\right) \mid 0 \epsilon v(\langle 1\rangle)\right) .
$$

By (42) and the definition of the transformations $T_{t}$ and $L_{\xi}$ we have

$$
T_{t} L_{\xi_{i}} A\left(\xi_{i}, k_{i}\right)=\left\{\sum_{\eta \in \Xi} \chi(v(\eta), T-t)=k_{i}\right\}=\left\{\omega(v, T-t)=k_{i}\right\}
$$

and, by property (a) of the function $v,\{0 \epsilon v(\langle 1\rangle)\}=\nabla$. Hence formula (45) may be written in the form

$$
P\left(A\left(\xi_{i}, k_{i}\right) \mid t \in v\left(\xi_{i}\right)\right)=P\left(\omega\left(v, T-t_{i}\right)=k_{i}\right) .
$$

Thus from formulae (43) and (44) we get

$$
\begin{aligned}
& P\left(\{\omega(v, T)=k\} \cap\{\omega(v, t)=n\} \cap \bigcap_{j=1}^{m}\left\{\omega\left(v, t_{j}\right)=n_{j}\right\}\right) \\
& =\left[\sum_{k_{1}+\ldots+k_{n}=k} \prod_{i=1}^{n} P\left(\omega(v, T-t)=k_{i}\right)\right] P\left(\bigcup_{*}^{n}\left\{t \in v\left(\xi_{i=1}^{n}\right)\right\} \cap B\left(\xi_{1}, \ldots, \xi_{n}\right)\right) ;
\end{aligned}
$$

this, together with equality (40), implies our lemma.

LEMMA 6. If $T>t$, then for every $k, n_{1}, \ldots, n_{m}, t_{1}, \ldots, t_{m}$ and $m \geqslant 0$ we have

$$
\begin{aligned}
P(\{\omega(v, T)=k\} \cap\{\omega(n, t) & \left.=0\} \cap \bigcap_{j=1}^{n}\left\{\omega\left(v, t_{j}\right)=n_{j}\right\}\right) \\
& =\delta_{0}^{k} P\left(\{\omega(v, t)=0\} \cap \bigcap_{j=1}^{m}\left\{\omega\left(v, t_{j}\right)=n_{j}\right\}\right) .
\end{aligned}
$$

Proof. It follows from the definition of the function $\omega$ and from the properties (a), (b), and (d) of the function $v$ for $T>t$ that

$$
\{\omega(v, t)=0\} \subset\{\omega(v, T)=0\} .
$$

Thus

$$
\{\omega(v, T)=k\} \cap\{\omega(v, t)=0\}=\left\{\begin{array}{ccc}
\{\omega(v, t)=0\} & \text { as } & k=0, \\
0 & \text { as } & k \neq 0
\end{array}\right.
$$

whence the lemma follows directly.

Lemma 7. For every $n$ we have

$$
\lim _{t \rightarrow T++}\{\omega(v, t) \geqslant n\}=\{\omega(v, T) \geqslant n\} .
$$

Proof. Suppose that $v_{0} \epsilon\{\omega(v, T) \geqslant n\}$. By definition (21) of the function $\omega$ we see that there exists a system $\xi_{1}, \ldots, \xi_{n}\left(\xi_{i} \in \Xi\right)$ such that (46)

$$
\xi_{i} \neq \xi_{j} \quad \text { if } \quad i \neq j
$$

and $T \in v_{0}\left(\xi_{i}\right)(i=1,2, \ldots, n)$.

Let $v_{0}\left(\xi_{i}\right)=\left[a_{i}, b_{i}\right) \quad(i=1,2, \ldots, n)$. Then for every $t$ satisfying the inequality

$$
T<t<\min _{1 \leqslant i \leqslant n} b_{i}
$$


we have the relation $t e v_{0}\left(\xi_{i}\right)(i=1,2, \ldots, n)$ which, in virtue of $(46)$, leads to $\omega\left(v_{0}, t\right) \geqslant n$. Thus we have proved that

$$
\{\omega(v, T) \geqslant n\} \subset \liminf _{t \rightarrow T^{+}}\{\omega(v, t) \geqslant n\} .
$$

Suppose now that

$$
v_{0} \in \limsup _{t \rightarrow T_{+}}\{\omega(v, t) \geqslant n\} .
$$

Then there exists a sequence of numbers

$$
t_{1}>t_{2}>\ldots \rightarrow T
$$

and a sequence of systems of elements of the set $\Xi$

such that

$$
\xi_{1, r}, \xi_{2, r}, \ldots, \xi_{n, r} \quad(r=1,2, \ldots)
$$

and

(49)

$$
\dot{\xi}_{i, r} \neq \xi_{j, r} \quad \text { for } \quad i \neq j, r=1,2, \ldots,
$$

$$
t_{r} \in v_{0}\left(\xi_{i, r}\right) \quad(i=1,2, \ldots, n ; r=1,2, \ldots) .
$$

By formulae (47) and (49)

$$
v_{0}\left(\xi_{i, r}\right) \frown\left[0, t_{1}\right) \neq 0 \quad(i=1,2, \ldots, n ; v=1,2, \ldots) ;
$$

whence from property $\left(\theta^{\prime}\right)$ it follows that among the systems $\xi_{1, r}, \xi_{2, r}, \ldots, \xi_{n, r}$ only a finite number is different. Thus there exists a subsequence $r_{1}, r_{2}, \ldots$ such that $\xi_{i, r}=\eta_{i}\left(i=1,2, \ldots, n ; r=r_{1}, r_{2}, \ldots\right)$ where, because of (48),

$$
\eta_{i} \neq \eta_{j} \quad \text { as } \quad i \neq j .
$$

Hence by (49) we infer that $t_{r} \in v_{0}\left(\eta_{i}\right)\left(i=1,2, \ldots, n ; r=r_{1}, r_{2}, \ldots\right)$, which, in view of (47), implies $T \in v_{0}\left(\eta_{i}\right)(i=1,2, \ldots, n)$.

Thus by formula $(50)$ we deduce that $\omega\left(v_{0}, T\right) \geqslant n$. Hence we have proved that

$$
\limsup _{t \rightarrow T+}\{\omega(v, t) \geqslant n\} \subset\{\omega(v, T) \geqslant n\},
$$

which, tegether with the inclusion proved before, leads to our lemma.

Corollary. By lemma 7 we infer that

$$
\lim _{t \rightarrow 0+}\{\omega(v, t)=1\}=\{\omega(v, 0)=1\}=V,
$$

which implies the equality

$$
\lim _{t \rightarrow 0+} P(\omega(v, \dot{t})=k)=\delta_{0}^{k} .
$$

Proof of Theorem 1. The space $\Omega(V)$ consists of step-functions defined on the positive semi-axis, taking on non-negative integral values and equal to 1 for $t=0$. Hence, $73_{\Omega(V)}$ being the smallest $\sigma$-field spanned on the sets of the form (1), in order to prove Theorem 1 it is sufficient to show that, for this process, there exist transition probabilities $\boldsymbol{P}_{n}^{k}(t)$ satisfying conditions (2), (3), (4), (5) and (14).

Let us write

(51) $. \quad P_{1}^{k}(t)=\Omega P\{\omega: \omega(t)=k\}=P(\omega(v, t)=k) \quad(k=0,1, \ldots)$,

(52) $P_{n}^{k}(t)=\sum_{k_{1}+\ldots+k_{n}=k} \prod_{i=1}^{n} P_{1}^{k_{i}}(t), P_{0}^{k}(t)=\delta_{0}^{k} \quad(n=1,2, \ldots ; k=0,1, \ldots)$.

Let $f_{n}(x, t)$ be the generating function of the sequence $P_{n}^{0}(t), P_{n}^{1}(t), \ldots$ :

By definition (52)

(54)

$$
f_{n}(x, t)=\sum_{k=0}^{\infty} P_{n}^{k}(t) x^{k}
$$

whence

$$
f_{n}(x, t)=\left[f_{1}(x, t)\right]^{n}
$$

$$
\sum_{k=0}^{\infty} P_{n}^{k}(t)=f_{n}(1, t)=\left[f_{1}(1, t)\right]^{n}=\left[\sum_{k=0}^{\infty} P(\omega(v, t)=k)\right]^{n}=1
$$

which gives formula (2), the functions $P_{n}^{k}(t)$ being non-negative.

Using definitions (51) and (52) we may write the conclusion of lemmas 5 and 6 in the following form:

$$
\begin{aligned}
P(\{\omega(v, T)= & \left.k\} \cap\{\omega(v, t)=n\} \cap \bigcap_{j=1}^{. m}\left\{\omega\left(v, t_{j}\right)=n_{j}\right\}\right) \\
& =P_{m}^{k}(T-t) P\left(\{\omega(v, t)=n\} \frown \bigcap_{j=1}^{m}\left\{\omega\left(v, t_{j}\right)=n_{j}\right\}\right)
\end{aligned}
$$

where $T>t>t_{1}>\ldots>t_{m}$. Applying repeatedly lemmas 5 and 6 to the second factor on the right side of formula (55), we obtain (5).

$$
\begin{aligned}
& \text { By (51) and (55) we get } \\
& P_{1}^{k}(T+t)=\sum_{s=0}^{\infty} P(\{\omega(v, T+t)=k\} \frown\{\omega(v, t)=s\})=\sum_{s=0}^{\infty} P_{s}^{k}(T) P_{1}^{s}(t) .
\end{aligned}
$$

Multiplying this equality by $x^{k}$ and summing up from $k=0$ to $\infty$ we get in virtue of (53)

$$
f_{1}(x, T+t)=\sum_{s=0}^{\infty} f_{s}(x, T) P_{1}^{s}(t)
$$

whence by (54) it follows that

$$
f_{1}(x, t+T)=\sum_{s=0}^{\infty}\left[\dot{f}_{1}(x, T)\right]^{s} P_{1}^{s}(t)=f_{1}\left(f_{1}(x, T), t\right) .
$$


Thus we get

$$
f_{n}(x, t+T)=\left[f_{1}(x, t+T)\right]^{n}=\left[f_{1}\left(f_{1}(x, T), t\right)\right]^{n}=f_{n}\left(f_{1}\left(x, T^{\prime}\right), t\right),
$$

i. e.,

$$
\begin{aligned}
\sum_{k=0}^{\infty} P_{n}^{k}(t+T) x^{k} & =\sum_{s=0}^{\infty}\left[f_{1}(x, T)\right]^{s} P_{n}^{s}(t)=\sum_{s=0}^{\infty} f_{s}(x, T) P_{n}^{s}(t) \\
& =\sum_{k=0}^{\infty}\left[\sum_{s=0}^{\infty} P_{s}^{k}(T) P_{n}^{s}(t)\right] x^{k}
\end{aligned}
$$

whence formula (3) follows.

The corollary of Lemma 7, and definitions (51) and (52) imply

$$
\lim _{t \rightarrow 0+} P_{n}^{k}(t)=\sum_{k_{1}+\ldots+k_{n=1} k_{i}} \prod_{i=1}^{n} \delta_{1}^{k_{i}}=\delta_{n}^{k}=P_{n}^{k}(0) ;
$$

condition (4) is thus fulfilled. Finally, definition (52) directly implies condition (14), which concludes the proof.

7. Suppose we are given a homogeneous regular Markov process $\left\langle\Omega, 73_{\Omega}\right.$, Pr $\rangle$ with a denumerable multitude of states, satisfying conditions (13) and (14). Let the numbers

$$
\pi_{1}^{0}, \pi_{1}^{1}, \pi_{1}^{2}, \ldots
$$

be the intensities of this process (the remaining ones are already defined by formula (15)). In order to prove Theorem 2 it is sufficient to show that there exists a process $\left\langle V, 7 \beta_{V}, P\right\rangle$ inducing the process $\left\langle\Omega(V),{ }_{\beta_{\Omega(V)}}, \Omega P\right\rangle$ with the intensities (56) (compare section 1).

Suppose that $\pi_{1}^{1}=0$; then for almost every $\omega \epsilon \Omega$ we have $\omega(t)=1$, for $\omega(0)=1$ (compare [2], p. 457). Defining the measure of the process $\left\langle V, 79_{v}, P\right\rangle$ by the formula

$$
P(A)=\left\{\begin{array}{lll}
1 & \text { as } & v_{0} \in A, \\
0 & \text { as } & v_{0} \notin A,
\end{array}\right.
$$

where $A \epsilon{ }_{Y}$ and $v_{0}(\langle 1\rangle)=[0, \infty), v_{0}(\xi)=0$ for $\xi \neq\langle 1\rangle$, we infer that for almost any $v \epsilon V$ the equality $\omega(v, t) \equiv 1$ is satisfied, whence the processes $\left\langle\Omega(V),{ } \beta_{\Omega(V)}, \Omega P\right\rangle$ and $\left\langle\Omega, C 3_{\Omega}, P r\right\rangle$ are identical, which provies Theorem 2 in the case in question. Thus it is sufficient to deal in the sequel with the case

$$
\pi_{1}^{1}>0 \text {. }
$$

Let $W$ be the space of all pairs $w=\left\langle w_{1}, w_{2}\right\rangle$ of functions defined on $g$, taking on non-negative real values including $\infty$. Every element $v \in V$ may be considered as belonging to $W$; to do this we define the pair $\left\langle w_{1}, w_{2}\right\rangle$ as

$$
\left.\begin{array}{l}
w_{1}(\xi)=a_{\xi} \\
w_{i}(\xi)=b_{\xi}
\end{array}\right\} \quad \text { when } \quad v(\xi)=\left[a_{\xi}, b_{\xi}\right) .
$$

Thus we have

$$
V \subset W
$$

Let $\mathfrak{B}_{W}$ be the smallest $\sigma$-field spanned upon the sets of form

$$
C(\xi, t, T)=\left\{w: w \epsilon W, w_{1}(\xi)<t, w_{-}(\xi)<T\right\} .
$$

Hence by the definition of the $\sigma$-field $\Upsilon_{V}$ and inclusion (58) we obtain

$$
\mathfrak{H}_{V}=\mathfrak{B}_{W} \cap V
$$

(compare [6], p. 25).

In the case (57) the proof consists of several parts. First, by aid of intensities (56) we define a normed $\sigma$-measure $P_{0}$ on the $\sigma$-field $\mathfrak{Z}_{T V}$; then we prove that the set $V$ is measurable and $P_{0}(V)=1$. Hence making use of the well-known relativization of the $\sigma$-measure $P_{0}$ to the set $V$ we obtain, in view of (60), a $\sigma$-measure $P$ defined on the $\sigma$-field $73_{\mathrm{V}}$. We prove that $P$ satisfies conditions (I), (II), and (III), whence we obtain a cascade process $\left\langle V, \cap_{V}, P\right\rangle$. Finally, we prove that the process $\left\langle\Omega(V),{ }^{\prime} \beta_{\Omega(V)}, \Omega P\right\rangle$ generated by this process has intensities (56), and this concludes the proof of Theorem 2.

8. The space $W$ may be considered as the product

$$
W=\underset{\xi \in \Xi}{P}\left(X_{\xi} \times Y_{\xi}\right)
$$

where the factors $\mathcal{X}_{\xi}$ and $\mathcal{Y}_{\xi}(\xi \in \Xi)$ are sets composed of non-negative real numbers including $\infty$.

Let $\mathfrak{Z}(Y)(Y \subset \Xi)$ denote the smallest $\sigma$-field spanned on the sets of form (59) where $\xi \in Y$. Thus $\mathfrak{Z}(Y)$ is the $\sigma$-field of cylinders of the product $(61)$, whose bases are Borel sets contained in the product

$$
\prod_{\xi \in Y}\left(X_{\xi} \times \mathscr{Y}_{\xi}\right)
$$

Let the set $Y$ consist of the elements $\xi_{1}, \xi_{2}, \ldots, \xi_{n}$. The function $F\left(t_{\xi_{1}}, t_{\xi_{2}}, \ldots, t_{\xi_{n}} ; T_{\xi_{1}}, T_{\xi_{2}}, \ldots, T_{\xi_{n}}\right)$ of $2 n$ real variables will be denoted simply as $F_{Y}\left(t_{\xi}, T_{\xi}\right)$. 

mulae:

We now define the operators $\Delta_{\xi_{i}}^{(1)}(h)$ and $\Delta_{\xi_{i}}^{(2)}(h)$ by the following for-

$$
\begin{array}{r}
\Delta_{\xi_{i}}^{(1)}(h) F_{Y}^{\prime}\left(t_{\xi}, T_{\xi}\right)=F\left(t_{\xi_{1}}, \ldots, t_{\xi_{i-1}}, t_{\xi_{i}}+h, t_{\xi_{i+1}}, \ldots, t_{\xi_{n}} ; T_{\xi_{1}}, \ldots, T_{\xi_{\xi_{n}}}\right) \ldots \\
-F\left(t_{\xi_{1}}, \ldots, t_{\xi_{n}} ; T_{\xi_{1}}, \ldots, T_{\xi_{n}}\right) \\
A_{\xi_{i}}^{(2)}(h) F_{Y}\left(t_{\xi}, T_{\xi}\right)=F^{\prime}\left(t_{\xi_{1}}, \ldots, t_{\xi_{n}} ; T_{\xi_{1_{1}}}, \ldots, T_{\xi_{i_{i-1}}}, T_{\xi_{i}}+h, T_{\xi_{i+1}}, \ldots, T_{\xi_{n}}\right)- \\
-F\left(t_{\xi_{1}}, \ldots, t_{\xi_{n}} ; T_{\xi_{\xi_{1}}}, \ldots, T_{\xi_{\eta_{n}}}\right)
\end{array}
$$

Let us write

$$
\Delta_{Y}\left(h_{\xi}, k_{\xi}\right)=\prod_{\xi \in \Gamma} \Delta_{\xi}^{(1)}\left(h_{\xi}\right) \Delta_{\xi}^{(2)}\left(k_{\xi}\right) .
$$

If the set $Y \subset \Xi$ is finite, then the distribution function in the product (62) (which is a closed Euclidean space with the number of dimensions equal to the doubled multitude of the set $X$ ) is the term given to every function $F_{Y}\left(t_{\xi}, T_{\xi}\right)$ satisfying the following conditions:

1. the function $F_{Y}\left(t_{\xi}, T_{\xi}\right)$ is continuous on the left with respect to every argument $t_{\xi}, T_{\xi}$;

2. for every argument $t_{\xi}, T_{\xi}$ and non-negative $h_{\xi}, k_{\xi}$ the inequality $\left.\Delta_{Y}\left(h_{\xi}, k_{\xi}\right) F_{Y}\left(t_{\xi}, T_{\xi}\right) \geqslant 0^{3}\right)$ holds.

3. $F_{Y}\left(t_{\xi}, T_{\xi}\right)=1$ as $t_{\xi}=T_{\xi}=\infty$ for $\xi \in Y, F_{Y}\left(t_{\xi}, T_{\xi}\right)=0$ as $t_{\xi} \leqslant 0$ or $T_{\xi} \leqslant 0$ for a certain $\xi \in Y$.

The class of all distribution functions in the product $(62)$ will be denoted by $D(Y)$. We quote here the well-known properties of the distribution functions:

$\left(\mathrm{d}_{1}\right)$ If $Y_{1} \cap Y_{2}=0, F_{1} \in \mathcal{D}\left(Y_{1}\right)$ and $F_{2} \in \mathcal{D}\left(Y_{2}\right)$, then $F_{1} \cdot F_{2} \in \mathcal{D}\left(Y_{1} \cup Y_{2}\right)$.

$\left(\mathrm{d}_{2}\right)$ If $F_{Y}\left(t_{\xi}, T_{\xi}\right) \in \mathcal{D}(\bar{Y})$, then $F_{Y}\left(t_{\xi}-t, T_{\xi}-t\right) \in \mathcal{D}(Y)$ for every $t \geqslant 0$.

$\left(\mathrm{d}_{3}\right)$ If $F_{t} \in \mathcal{D}(Y)$ for $t \geqslant 0$ and if $f(t)$ is a distribution function on the semi-axis $t \geqslant 0$, then

$$
\int_{0}^{\infty} F_{t} d f(t) \epsilon \mathscr{D}(Y)
$$

This implies in particular that

$\left(\mathrm{d}_{4}\right)$ Every convex combination of distribution functions is a distribution function: if $F_{1}, \ldots, F_{n} \in \mathcal{D}(Y), \lambda_{1} \geqslant 0, \ldots, \lambda_{n} \geqslant 0$ and $\lambda_{1}+\ldots+\lambda_{n}=1$, then $\lambda_{1} F_{1}+\ldots+\lambda_{n} F_{n} \in \mathcal{D}(Y)$.

If $\xi=\left\langle x_{1}, \ldots, x_{n}\right\rangle$, then the integer $n$ is called the length of the element $\xi$. The length of a finite set is defined as the maximal length of its elements.

3) $\Delta_{Y}\left(h_{\xi}, k_{\xi}\right) F_{Y}\left(t_{\xi}, T_{\xi}\right)$ denotes the volume of the eube ${ }_{\xi}\left(\left[t_{\xi}, t_{\xi}+h_{\xi}\right) \times\right.$
9. For every finite set $Y \subset \Xi$ we shall define, by aid of intensities (56), a distribution function $\nu_{Y}\left(t_{\xi}, T_{\xi}\right) \in \mathcal{D}(\bar{Y})$. This will be done by induction with respect to the length of the set $Y$.

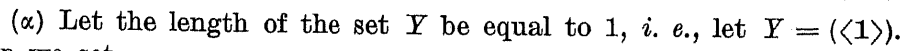
Then we set

$$
v_{Y}\left(t_{\xi}, T_{\xi}\right)=\left\{\begin{array}{cl}
1-\exp \left(-\pi_{1}^{1} T_{\langle 1\rangle}\right) & \text { as } t_{\langle 1\rangle}>0 \text { and } T_{\langle 1\rangle}>0, \\
0 & \text { elsewhere. }
\end{array}\right.
$$

It is easily verified that conditions 1,2 , and $3($ p. 252$)$ are verified, whence $\nu_{Y}\left(t_{\xi}, T_{\xi}\right) \in \mathbb{D}((\langle 1\rangle))$.

( $\beta$ ) Suppose now that the length of the set $Y$ is greater than 1 and that the distribution function $\nu_{T}\left(t_{\xi}, T_{\xi}\right)$ is already defined for all sets with smaller lengths.

Let us consider first the case

$$
\langle 1\rangle \notin Y \text {. }
$$

Then the set $Y$ may be represented as the union of disjoint summands

where

$$
Y=\bigcup_{j} \bigcup_{i} Y_{i, i}
$$

$$
Y_{i, j}=Y_{\bigcap} K(\langle 1,\langle i, j\rangle\rangle) .
$$

It follows from (65) that $\xi \in Y_{i, j}$ implies $\xi-\langle 1,\langle i, j\rangle\rangle \in \xi$. For non-void $Y_{i, j}$ let us put

$$
Y_{i, j}^{*}=\left\{\xi-\langle 1,\langle i, j\rangle\rangle: \xi \in Y_{i, j}\right\} .
$$

We see from this formula that the length of the set $Y_{i, j}^{*}$ is less than that of $Y$.

Let $\varrho_{Z}\left(t_{\xi}, T_{\xi}\right)$ be the unit distribution, $i$. e., let

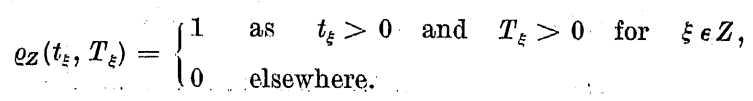

In the case $(64)$ the distribution function $v_{Y}\left(t_{\xi}, T_{\xi}\right)$ will be defined by the formula

(67)

$$
\begin{aligned}
& \nu_{Y}\left(t_{\xi}, T_{\xi}\right) \\
& =\sum_{j \in M} \pi_{1}^{j} \int_{0}^{\infty} \exp \left(-\pi_{1}^{1} t\right) \varrho_{Y-Y_{i} Y_{i, j}}\left(t_{\xi}-t, T_{\xi}^{1}-t\right) \prod \prod_{i} v_{Y_{i, j}^{*}}\left(t_{\xi}-t, T_{\xi}-t\right) d t+ \\
& +\left(\pi_{1}^{1}-\sum_{j \in M} \pi_{1}^{j}\right) \int_{0}^{\infty} \exp \left(-\pi_{1}^{1} t\right) \varrho_{Y}\left(t_{\xi}-t, T_{\xi}-t\right) d t,
\end{aligned}
$$


where $M=\left\{j: \bigcup_{i} Y_{i, j} \neq 0\right\}$. In this formula we consider only non-void summands $Y_{i, j}$, whence in view of (66) only non-void sets $Y_{i, j}^{*}$. From the hypothesis of induction it follows that the distribution functions $\nu_{X_{i, j}^{*}}\left(t_{\xi}, T_{\xi}\right)$ are already defined for $Y_{i, j}^{*} \neq 0$, since the length of $Y_{i, j}^{*}$ is less that that of $Y$. It follows from formula (66) that changing suitably the numbering of the variables we may consider $v_{Y_{i, j}^{*}}\left(t_{\xi}, T_{\xi}\right)$ as an element of $\mathcal{D}\left(Y_{i, j}\right)$. The sets $Y_{i, j}, \ldots, Y_{j, j}, Y-\bigcup_{i} Y_{i, j}$ being disjoint, we infer by $\left(d_{1}\right)$ and $\left(d_{2}\right)$ that for $t \geqslant 0$

(68) $\quad F_{j}(t)=\varrho_{Y-\bigcup Y_{i, j}}\left(t_{\xi}-t, T_{\xi}-t\right) \prod_{i} v_{X_{i, j}^{*}}\left(t_{\xi}-t, T_{\xi}-t\right) \epsilon(D)(Y), \quad j \in M$.

From inequality (57) we deduce that the function $f(t)=1-\exp \left(-\pi_{1}^{1} t\right)$ is a distribution function on the semi-axis $t \geqslant 0$, whence by $\left(d_{3}\right)$ and $(68)$ we get

$$
\begin{aligned}
& G_{Y}^{j}\left(t_{\xi}, T_{\xi}\right)=\pi_{1}^{1} \int_{0}^{\infty} \exp \left(-\pi_{1}^{1} t\right) F_{j}(t) d t=\int_{0}^{\infty} F_{j}(t) d f(t) \epsilon(D(Y) \quad(j \in M), \\
& C_{Y}^{0}\left(t_{\xi}, T_{\xi}\right)=\pi_{1}^{1} \int_{0}^{\infty} \exp \left(-\pi_{1}^{1} t\right) \varrho_{Y}\left(t_{\xi}-t, T_{\xi}-t\right) d t \epsilon D(Y) .
\end{aligned}
$$

Setting $\lambda_{i}=\pi_{1}^{i} / \pi_{1}^{1}$ for $j \in M$, and

$$
\lambda_{0}=1-\sum_{j \in M} \pi_{1}^{j} / \pi_{1}^{1}
$$

we have $\lambda_{j} \geqslant 0(j \in M), \lambda_{0} \geqslant 0$, and

$$
\lambda_{0}+\sum_{j \in M} \lambda_{j}=1
$$

(we take into account the inequality $j \geqslant 2$ valid for $j \in M$ and formulae (8) and (9)). By aid of $\left(d_{4}\right)$ and formulae $(67),(68),(69)$, and (70) we get

$$
\nu_{Y}\left(t_{\xi}, T_{\xi}\right)=\lambda_{0} G_{Y}^{0}+\sum_{j \in M} \lambda_{j} G_{Y}^{j} \in \mathscr{D}(Y),
$$

whence formula (67) defines a distribution function in the case (64). From (67) we deduce

$$
\begin{aligned}
& \Delta_{Y}\left(h_{\xi}, k_{\xi}\right) v_{Y}\left(t_{\xi}-T, T_{\xi}-T\right) \\
& =\sum_{j} \pi_{1}^{j} \int_{0}^{\infty} \exp \left(-\pi_{1}^{1} t\right) \Delta_{Y-U Y_{i, j}}\left(h_{\xi}, k_{\xi}\right) \varrho_{Y-U Y_{i, j}}\left(t_{\xi}-(T+t), T_{\xi}-(I+t)\right) \times \\
& \quad \times \prod_{i} \Delta_{Y_{i, j}^{*}, j}\left(h_{\xi}, k_{\xi}\right) v_{Y_{i, j}^{*}}\left(t_{\xi}-(T+t), T_{\xi}-(T+t)\right) d t+ \\
& \quad+\left(\pi_{1}^{1}-\sum_{j} \pi_{1}^{j}\right) \int_{0}^{\infty} \exp \left(-\pi_{1}^{1} t\right) \Delta_{Y}\left(h_{\xi}, k_{\xi}\right) \varrho_{Y}\left(t_{\xi}-(T+t), T_{\xi}-(T+t)\right) d t
\end{aligned}
$$

Replacing in the integrals on the right side of the last formula $T+t$ by a new variable we get, after simple computations,

$$
\Delta_{Y}\left(h_{\xi}, k_{\xi}\right) \nu_{Y}\left(t_{\xi}-T, T_{\xi}-T\right) \leqslant \exp \left(\pi_{1}^{1} T\right) \cdot \Delta_{F}\left(h_{\xi}, k_{\xi}\right) \nu_{Y}\left(t_{\xi}, T_{\xi}\right)
$$

for the sets satisfying condition (64).

Suppose now that

$$
Y=Z \cup(\langle 1\rangle) \quad \text { where } \quad\langle 1\rangle \notin Z .
$$

In this case we set

(73) $\quad v_{Y}\left(t_{\xi}, T_{\xi}\right)=v_{Z}\left(t_{\xi}, T_{\xi}\right)-\exp \left(-\pi_{1}^{1} T_{\langle 1\rangle}\right) v_{Z}\left(t_{\xi}-T_{\langle 1\rangle}, T_{\xi}-T_{\langle 1\rangle}\right)$

if $t_{\langle 1\rangle}>0$ and $T_{\langle 1\rangle}>0$; in the remaining cases we set $\nu_{Y}\left(t_{\xi}, T_{\xi}\right)=0$ The distribution function $v_{Z}\left(t_{\xi}, t_{\xi}\right)$ is already defined, for the set $Z$ satisfies condition (64). Hence follows also the continuity on the left in each variable of the function defined by (73). From (73) for $t_{\langle 1\rangle}>0$ and $T_{\langle 1\rangle}>0$ we deduce the equality

$$
\begin{aligned}
& \Delta_{Y}\left(h_{\xi}, k_{\xi}\right) v_{Y}\left(t_{\xi}, T_{\xi}\right)=\exp \left(-\pi_{1}^{1} T_{\langle 1\rangle}\right) \Delta_{Z}\left(h_{\xi}, k_{\xi}\right) v_{Z}\left(t_{\xi}-T_{\langle 1\rangle}, T_{\xi}-T_{\langle 1\rangle}\right)- \\
& -\exp \left(-\pi_{1}^{1}\left(T_{\langle 1\rangle}+k_{\langle 1\rangle}\right)\right) \Delta_{Z}\left(h_{\xi}, k_{\xi}\right) v_{Z}\left(t_{\xi}-\left(T_{\langle 1\rangle}+k_{\langle 1\rangle}\right), T_{\xi}-\left(T_{\langle 1\rangle}-k_{\langle 1\rangle}\right)\right),
\end{aligned}
$$

for $t_{\langle 1\rangle}>0, T_{\langle 1\rangle}+k>0$ and $T_{\langle 1\rangle} \leqslant 0$ the equality

$$
\begin{aligned}
& \Delta_{Y}\left(h_{\xi}, k_{\xi}\right) v_{Y}\left(t_{\xi}, T_{\xi}\right)=\Delta_{Z}\left(h_{\xi}, k_{\xi}\right) v_{Z}\left(t_{\xi}, T_{\xi}\right)- \\
& -\exp \left(-\pi_{1}^{1}\left(T_{\langle 1\rangle}+k_{\langle 1\rangle}\right)\right) \Delta_{Z}\left(h_{\xi}, k_{\xi}\right) v_{Z}\left(t_{\xi}-\left(T_{\langle 1\rangle}+t_{\langle 1\rangle}\right), T_{\xi}-\left(T_{\langle 1\rangle}+t_{\langle 1\rangle}\right)\right),
\end{aligned}
$$

and in the remaining cases $\Delta_{Y}\left(h_{\xi}, k_{\xi}\right) \nu_{Y}\left(t_{\xi}, T_{\xi}\right)=0$.

Inequality (71) being true for the set $Z$, we obtain for arbitrary $t_{\xi}, T_{\xi}$ and non-negative $h_{\xi}, k_{\xi}$

$$
\Delta_{Y}\left(h_{\xi}, k_{\xi}\right) \nu_{Y}\left(t_{\xi}, T_{\xi}\right) \geqslant 0 .
$$

We see from (73) that $v_{Y}\left(t_{\xi}, T_{\xi}\right)=v_{Z}\left(t_{\xi}, T_{\xi}\right)$ for $t_{\xi}=T_{\xi}=\infty, \xi \epsilon Y$. Hence $v_{Y}\left(t_{\xi}, T_{\xi}\right)=1$ if $t_{\xi}=T_{\xi}=\infty$ for $\xi \in Y$. If $t_{\xi} \leqslant 0$ or $T_{\xi} \leqslant 0$ for a certain $\xi \in Z$, then $v_{Z}\left(t_{\xi}, T_{\xi}\right)=0$ and $\nu_{Z}\left(t_{\xi}-T, T_{\xi}-T\right)=0$ for $T>0$, which together with (73) leads to $\nu_{Y}\left(t_{\xi}, T_{\xi}\right)=0$ if $t_{\xi} \leqslant 0$ or $T_{\xi} \leqslant 0$ for a certain $\xi \in Y$. Thus we have shown that formula (73) defines a distribution function, which completes the inductive definition of $v_{Y}\left(t_{\xi}, T_{\xi}\right)$ for all $\boldsymbol{Y}$. By definitions (63), (67), and (73) it is easily seen that for $Y \cap Z=0$ the fol lowing compatibility condition is satisfied:

$$
\nu_{Y \cup Z}\left(t_{\xi}, T_{\xi}\right)=\nu_{Y}\left(t_{\xi}, T_{\xi}\right) \quad \text { if } \quad t_{\xi}=T_{\xi}=\infty \text { for } \xi \in Z .
$$

The distribution function $\nu_{Y}\left(t_{\xi}, T_{\xi}\right)$ determines in the product (62) a normed $\sigma$-measure $\mu_{Y}$ defined on all Borel sets, $i$. e., on the sets of the $\sigma$-field $\mathfrak{Z}(Y)$. By the compatibility condition (74), $A \epsilon \mathfrak{B}(Y) \cap \mathfrak{B}(Z)$ implies 
$\mu_{Y}(A)=\mu_{Z}(A)$. Setting $\mu(A)=\mu_{Y}(A)$ when $A \epsilon \mathfrak{B}(Y)$, we define a function $\mu$ on the field of cylinders whose bases are finite dimensional Borel sets, $i$. e., on

$$
\bigcup_{Y} \mathfrak{Z}(Y)
$$

where $Y$ are finite subsets of $\Xi$. The function $\mu$ is, by definition, a $\sigma$-mearsure on every $\mathfrak{Z}(Y)$ ( $Y$ is a finite set), and $\mu(W)=1$.

By a theorem of Kolmogorov (see, for instance, [6], p. 212) it follows that there exists a unique extension of the function $\mu$ to a normed $\sigma$-measure, which will be denoted by $P_{0}$; this measure is defined on the smallest $\sigma$-field containing the field

$$
\bigcup_{Y} \mathfrak{Z}(\bar{Y})
$$

of the finite dimensional cylinders, whence on the $\sigma$-field $\mathfrak{Z}_{W}$ (compare section 7).

By the definition of $P_{0}$ and by formulae $(63),(67)$, and (73) we get

$$
\begin{aligned}
& \text { (75) } \\
& P_{0}\left(w_{1}(\langle 1\rangle)=a, w_{2}(\langle 1\rangle)<T\right)=\left\{\begin{array}{cc}
1-\exp \left(-\pi_{1}^{1} T\right) & \text { if } T>0 \text { and } a= \\
0 & \text { elsewhere, }
\end{array}\right. \\
& \begin{aligned}
\text { (76) } \quad P_{0}\left(\bigcap_{\xi \in Y}\left\{w_{1}(\xi)<t_{\xi}, w_{2}(\xi)<T_{\xi}\right\} \cap\left\{w_{2}(\langle 1\rangle)<T\right\}\right) \\
=P_{0}\left(\bigcap_{\xi \in Y}\left\{w_{1}(\xi)<t_{\xi}, w_{2}(\xi)<T_{\xi}\right\}\right)- \\
\quad-\exp \left(-\pi_{1}^{1} T\right) P_{0}\left(\bigcap_{\xi \in Y}\left\{w_{1}(\xi)<t_{\xi}-T, w_{2}(\xi)<T_{\xi}-T\right\}\right)
\end{aligned}
\end{aligned}
$$

for $T>0$ and the sets $Y$ satisfying condition (64),

$$
\begin{aligned}
& \text { (77) } \quad P_{0}\left(\bigcap_{\xi \in Y}\left\{w_{1}(\xi)<t_{\xi}, w_{2}(\xi)<T_{\xi}\right\}\right) \\
& =\sum_{j} \pi_{1}^{j} \int_{0}^{\infty} \exp \left(-\pi_{1}^{1} t\right) \delta\left(\bigcap_{\xi \in Y-\bigcup_{i} Y_{i, j}}\left\{w_{1}(\xi)<t_{\xi}-t, w_{2}(\xi)<T_{\xi}-t\right\}\right) \times \\
& \times \prod_{i} P_{0}\left(\bigcap_{\xi \in Y_{i, j}}\left\{w_{1}(\xi-\langle 1,\langle i, j\rangle\rangle)<t_{\xi}-t, w_{2}(\xi-\langle 1,\langle i, j\rangle\rangle)<T_{\xi}-t\right\}\right) d t+ \\
& \quad+\left(\pi_{1}^{1}-\sum_{j} \pi_{1}^{j}\right) \int_{0}^{\infty} \exp \left(-\pi_{1}^{1} t\right) \delta\left(\bigcap_{\xi \in Y}\left\{w_{1}(\xi)<t_{\xi}-t, w_{2}(\xi)<l_{\xi}^{1}-t\right\}\right) d t
\end{aligned}
$$

for the sets $Y$ satisfying (64). The sets $Y_{i, j}$ are defined by formula $(65)$ and the $\sigma$-measure $\delta$ by

$$
\delta(A)=\left\{\begin{array}{lll}
1 & \text { as } & w^{0} \in A, \\
0 & \text { as } & w^{0} \notin A,
\end{array}\right.
$$

where $w^{0}=\left\langle w_{1}^{0}, w_{2}^{0}\right\rangle$ is a pair of two functions identically equal to 0 . The following formulae define the transformations $\tau_{t}$ and $\mathcal{L}_{\xi}$ of the space $W$ :

(78) $\quad \tau_{t} w=\left\langle u_{1}, u_{2}\right\rangle$ where $\quad u_{1}(\xi)=w_{1}(\xi)-t, u_{2}(\xi)=w_{2}(\xi)-t$;

(79) $\quad \mathcal{L}_{\xi} w=\left\langle u_{1}, u_{2}\right\rangle$ where $u_{1}(\eta-\xi)=w_{1}(\eta), u_{2}(\eta-\xi)=w_{2}(\eta)$

for $\eta \in T(\xi)$.

Lemina 8. If $A \in \mathfrak{B}(\Xi-(\langle 1\rangle))$, then

(80) $\quad P_{0}\left(A \cap\left\{w_{2}(\langle 1\rangle)<T\right\}\right)=P_{0}(A)-\exp \left(-\pi_{1}^{1} T\right) P_{0}\left(\tau_{T} A\right)$

for $T>0$.

Proof. Let $\Omega$ be the class of all the sets $A$ belonging to the $\sigma$-field $\mathfrak{Z}(\Xi-(\langle 1\rangle))$ and satisfying our Lemma. By formula (76) we see that $Y \subset \Xi-(\langle 1\rangle)$ implies

$$
\bigcap_{\xi \in Y}\left\{t_{\xi}^{(1)} \leqslant w_{1}(\xi)<t_{\xi}^{(2)}, T_{\xi}^{(1)} \leqslant w_{2}(\xi)<T_{\xi}^{(2)}\right\} \in \Re .
$$

It follows that the finite disjoint unions of the sets of form (81) belong to the class $\mathfrak{R}$, whence $\mathfrak{R}$ contains the smallest field spanned upon the sets $(59)$ for $\xi \neq\langle 1\rangle$. From $(80)$ we directly deduce that the limit of a monotone sequence of sets of the class $\Omega$ also belongs to the class $\Omega$. Thus the class $\Omega$ is monotone (compare, for instance, [6], p. 26). Hence it follows by the definition of the $\sigma$-field $\mathfrak{Z}(\xi-(\langle 1\rangle))$ that $\mathfrak{R}=\mathfrak{Z}(\xi-(\langle 1\rangle))$ (compare [6], p. 27), which implies the lemma.

LEMMA 9. If $A_{i j} \in \mathfrak{B}(K(\langle 1,\langle i, j\rangle\rangle))$, then

$$
\begin{aligned}
& P_{0}\left(\bigcap_{j \in M} \bigcap_{i} A_{i j}\right)=\sum_{j \in M} \pi_{1}^{j} \int_{0}^{\infty} \exp \left(-\pi_{1}^{1} t\right) \delta\left(\bigcap_{s \neq j} \bigcap_{i} \tau_{t} A_{i s}\right) \times . \\
\times & \prod_{i} P_{0}\left(\tau_{t} \mathcal{L}_{\langle 1,\langle i, j\rangle\rangle} A_{i j}\right) d t+\left(\pi_{1}^{1}-\sum_{j \in M} \pi_{1}^{j}\right) \int_{0}^{\infty} \exp \left(-\pi_{1}^{1} t\right) \delta\left(\tau_{t} \bigcap_{i, j} A_{i j}\right) d t .
\end{aligned}
$$

The proof of this lemma is similar to the proof of the foregoing one. Formula (77) implies equality (82) for the sets $A_{i j}$ of form (81). Thus (82) is valid for finite disjoint unions of the sets of form (81). Then we verify that the class of the sets $A_{i j}$ (depending, of course, on $i$ and $j$ ) satisfying the lemma is monotone, whence it is equal to $\mathfrak{Z}(K(\langle 1,\langle i, j\rangle\rangle))$, which concludes the proof.

10. We shall now prove some lemmas which imply the measurability of the set $V$ together with the equality $P_{0}(V)=1$.

It follows directly from (75) that the set

$$
V_{1}=\left\{w_{1}(\langle 1\rangle)=0, w_{2}(\langle 1\rangle)>0\right\}
$$

Studia Mathemation XVI. 
is of full measure:

$(84)$

$$
P_{0}\left(V_{1}\right)=1 .
$$

This immediately implies

$$
P_{0}\left(w_{2}(\langle 1\rangle) \neq w_{1}(\langle 1,\langle r, m\rangle\rangle)\right)=0 .
$$

We obtain from (77) by aid of formulae (86) and (87) $2, \ldots, i$, let us introduce the following notation:

(85) $A_{m, r, s, n, k}(\xi)$

$=\left\{w_{2}(\xi)<w_{1}(\xi)\right\} \cup\left\{w_{1}(\xi)=w_{2}(\xi)<w_{2}\left(\langle\xi, r, m\rangle \cup\left\{w_{2}(\xi) \neq w_{1}(\langle\xi, r, m\rangle)\right\}\right) \cup\right.$ $\cup\left\{w_{2}(\langle\xi, r, m\rangle)>w_{1}(\langle\xi, r, m\rangle), w_{2}(\langle\xi, s, m\rangle)=w_{1}(\langle\xi, s, m\rangle)\right\} \cup$

$\left.\cup\left\{w_{2}(\langle\xi, r, m\rangle)>w_{1}(\langle\xi, r, m\rangle), w_{2}(\langle\xi, k, n\rangle)\right\rangle w_{1}(\langle\xi, k, n\rangle)\right\}$,

where $\langle\xi, r, m\rangle$ denotes the element $\left\langle x_{1}, \ldots, x_{n},\langle r, m\rangle\right\rangle$ for $\xi=:$ $=\left\langle x_{1}, \ldots, x_{n}\right\rangle$. The definitions of the transformations $\tau_{\text {, and }} \mathcal{L}_{\eta}$ imply

$$
\begin{aligned}
\tau_{t} A_{m, r, s, n, k} & =A_{m, r, s, n, k}(\xi), \\
\mathcal{L}_{\eta} A_{m, r, s, n, k} & =A_{m, r, s, n, k}(\xi-\eta) \quad \text { for } \quad \xi \in K(\eta) .
\end{aligned}
$$

LeMma 10. Fror every $\xi \epsilon \Xi$ we have $P_{0}\left(A_{m, r, \beta, n, k}(\xi)\right)=0$.

Proof. We shall prove this by induction with respect to the length of the element $\xi$.

( $\alpha$ ) Let the length of the element $\xi$ be equal to $1, i$. e., $\xi=\langle 1\rangle$. From (83) and (84) we infer that

$$
\begin{gathered}
P_{0}\left(w_{2}(\langle 1\rangle)<w_{1}(\langle 1\rangle)\right)=0, \\
P_{0}\left(w_{1}(\langle 1\rangle)=w_{2}(\langle 1\rangle)<w_{2}(\langle 1,\langle r, m\rangle\rangle)\right)=0 .
\end{gathered}
$$

Inserting $\left.A=\left\{w_{1}(\langle 1,\langle r, m\rangle\rangle)\right\rangle t\right\}$ in formula (80) we obtain

$P_{0}\left(w_{2}(\langle 1\rangle)<t<w_{1}(\langle 1,\langle r, r\rangle\rangle)\right)$

$$
\left.=P_{0}\left(w_{1}(\langle 1,\langle r, m\rangle\rangle)\right\rangle t\right)-\exp \left(-\pi_{1}^{1} t\right) P_{0}\left(w_{1}(\langle 1,\langle r, m\rangle\rangle)>0\right) .
$$

It follows from formula (82) with $\left.A_{r m}=\left\{w_{1}(\langle 1,\langle r, m\rangle\rangle)\right\rangle T\right\}$ $(T \geqslant 0), M=\{m\}, i=r$, and from (75) that

$$
\begin{aligned}
P_{0}\left(w_{1}(\langle 1,\langle r, m\rangle\rangle)>T\right) & =\pi_{1}^{m} \int_{0}^{\infty} \exp \left(-\pi_{1}^{1} t\right) P_{0}\left(w_{1}(\langle 1\rangle)>T-t\right) d t \\
& =\pi_{1}^{m} \int_{0}^{\infty} \exp \left(-\pi_{1}^{1} t\right) d t=\frac{\pi_{1}^{m}}{\pi_{1}^{1}} \exp \left(-\pi_{1}^{1} T\right),
\end{aligned}
$$

whence

$$
P_{0}\left(w_{2}(\langle 1\rangle)<t<w_{1}(\langle 1,\langle r, m\rangle\rangle)\right)=\frac{\pi_{1}^{m}}{\pi_{1}^{1}} \exp \left(-\pi_{1}^{1} t\right)-\frac{\pi_{1}^{m}}{\pi_{1}^{1}} \exp \left(-\pi_{1}^{1} t\right)=0 .
$$

$\left.P_{0}\left(w_{2}(\langle 1,\langle r, m\rangle\rangle)\right\rangle w_{1}(\langle 1,\langle r, m\rangle\rangle), w_{2}(\langle 1,\langle s, m\rangle\rangle)=w_{1}(\langle 1,\langle s, m\rangle\rangle)\right)$ $=\pi_{1}^{m} \int_{0}^{\infty} P_{0}\left(w_{2}(\langle\mathbf{1}\rangle)>w_{2}(\langle 1\rangle)\right) P_{0}\left(w_{2}(\langle 1\rangle)=w_{1}(\langle 1\rangle)\right) \exp \left(-\pi_{1}^{1} t\right) d t=0$,

$\left.P_{0}\left(w_{2}(\langle 1,\langle r, m\rangle\rangle)>w_{1}(\langle 1,\langle r, m\rangle\rangle), w_{2}(\langle 1,\langle k, n\rangle\rangle)\right\rangle w_{1}(\langle 1,\langle k, n\rangle\rangle)\right)$

$$
\begin{aligned}
= & \pi_{1}^{m} \int_{0}^{\infty} P_{0}\left(w_{2}(\langle 1\rangle)>w_{1}(\langle 1\rangle)\right) \delta\left(w_{2}(\langle 1,\langle k, n\rangle\rangle)\right. \\
> & \left.w_{1}(\langle 1,\langle k, n\rangle\rangle)\right) \exp \left(-\pi_{1}^{1} t\right) d t+ \\
& +\pi_{1}^{m} \int_{0}^{\infty} P_{0}\left(w_{2}(\langle 1\rangle)>w_{1}(\langle 1\rangle)\right) \delta\left(w_{2}(\langle 1,\langle r, m\rangle\rangle)\right. \\
> & \left.w_{1}(\langle 1,\langle r, m\rangle\rangle)\right) \exp \left(-\pi_{1}^{1} t\right) d t=0,
\end{aligned}
$$

whence in view of formulae (88), (89), and (90) we obtain our Lemma.

$$
\xi=\langle 1,\langle i, j\rangle, \ldots\rangle \neq\langle 1\rangle
$$

and that our Lemma is valid for elements of length less than that of $\xi$. It follows from (91) that

$$
A_{m ; r, s, n, k}(\xi) \epsilon \mathfrak{Z}(K(\langle 1,\langle i, j\rangle)))
$$

whence in virtue of formulae (82), (86), and (87)

$$
\begin{aligned}
P_{0}\left(A_{m, r, s, n, k}(\xi)\right)=\pi_{1}^{j} \int_{0}^{\infty} & P_{0}\left(A_{m, r, s, n, k}(\xi-\langle 1,\langle i, j\rangle\rangle)\right) \exp \left(-\pi_{1}^{1} t\right) d t+ \\
& +\left(\pi_{1}^{1}-\pi_{1}^{j}\right) \int_{0}^{\infty} \delta\left(A_{m, r, s, n, k}(\xi)\right) \exp \left(-\pi_{1}^{1} t\right) d t .
\end{aligned}
$$

By the hypothesis of induction

$$
P_{0}\left(A_{m, r, s, n, k}(\xi-\langle 1,\langle i, j\rangle\rangle)\right)=0,
$$

since the length of element $\xi-\langle 1,\langle i, j\rangle\rangle$ is less than the length of $\xi$. By the definition of the set $A_{m, r, s, n, k}(\xi)$ and the measure $\delta$ we also have $\delta\left(A_{m, r, s, n, k}(\xi)\right)=0$, which, in view of (92) and (93), implies the validity of the lemma for the element $\xi$. This concludes the proof.

\section{Corollary. Let us write}

$$
A=\bigcup_{\xi \in \Xi} \bigcup_{m=2} \bigcup_{\substack{n=2 \\ n \neq m}} \bigcup_{k=1}^{n} \bigcup_{r=1}^{m} \bigcup_{\substack{s=1 \\ s \neq r}}^{m} A_{m, r, s, n, k}(\xi)
$$

(B) Suppose now that 
We may easily verify from (85) that the set $A$ consists of functions $w$ which do not satisfy conditions (b), (c), or (d) (compare section 3). It follows by lemma 10 that

$$
P_{0}(A)=0
$$

Let us now recollect the well-known properties of homogeneous regular Markov processes with a denumerable multitude of states, satisfying conditions (13) and (14), i. e., of the processes describing a stochastic cascade. Suppose that the intensities are determined by formula (56).

Let $Q_{k}(n, T)$ be the probability that the function $\omega(t)$ (the realization of the process) has $n$ jumps in an interval of length $T$, under the hypothesis that at the origin of the interval the function $\omega(t)$ is equal to $k$. Then we have

$$
\begin{aligned}
Q_{k}(0, T) & =\exp \left(-\pi_{k}^{k} T\right), \\
Q_{k}(1, T) & =\sum_{j \neq k} \pi_{k}^{j} \int_{0}^{T} \exp \left(-\pi_{k}^{k} t\right) \exp \left(-\pi_{j}^{j}(T-t)\right) d t, \\
Q_{k}(n+1, T) & =\sum_{j \neq k} \pi_{k}^{j} \int_{0}^{T} \exp \left(-\pi_{k}^{k} t\right) Q_{j}(n, T-t) d t
\end{aligned}
$$

(compare [2], p. 458; for more general processes compare [3], p. 268).

For the processes satisfying condition (14) we have

$$
Q_{j}(n, T)=\sum_{n_{1}+\ldots+n_{j}=n} \prod_{i=1}^{j} Q_{1}\left(n_{i}, t\right)
$$

(this equality follows for $n=0$ from (15); the general proof proceeds by induction). Inserting $k=1$ in formulae (96) and taking into account that $Q_{0}(n, t)=0$ for $n \neq 0$ we obtain in view of (97)

(98) $\quad Q_{1}(0, T)=\exp \left(-\pi_{1}^{1} T\right)$,

$$
\text { (99) } \quad Q_{1}(1, T)=\sum_{j \neq 1} \pi_{1}^{j} \int_{0}^{T} \exp \left(-\pi_{1}^{1} t\right) \exp \left(-j \pi_{1}^{1}(T-t)\right) d t
$$

(100) $Q_{1}(n+1, T)$

$$
=\sum_{j=2}^{\infty} \pi_{1}^{j} \int_{0}^{T} \exp \left(-\pi_{1}^{1} t\right)\left[\sum_{n_{1}+\ldots+n_{j=n}} \prod_{i=1}^{j} Q_{1}\left(n_{i}, T-t\right)\right] d t \quad \text { for } \quad n \geqslant 1 .
$$

The regular processes satisfy the equality

$$
\sum_{n=0}^{\infty} Q_{1}(n, T)=1 \quad\left(T^{\prime} \geqslant 0\right)
$$

for the space of the process consists of step functions.
For every positive integer $n$ and $T \geqslant 0$ write

(102) $B_{n}(T)=\bigcup_{\xi_{1}, \ldots, \xi_{n} \in \Xi} \bigcap_{r=1}^{n}\left\{w_{1}\left(\xi_{r}\right)<w_{2}\left(\xi_{r}\right)<T\right\} \frown$

$$
\sim \bigcup_{\eta \neq \xi_{1}, \ldots, \xi_{n}}\left[\left\{w_{1}(\eta)=w_{2}(\eta)\right\} \cup\left\{w_{2}(\eta) \geqslant T\right\}\right],
$$

$$
V_{2}=\bigcap_{k=1}^{\infty} \bigcup_{n=0}^{\infty} B_{n}(k) \text {. }
$$

We see that, if the function $w$ satisfies conditions (a), (b), (c), and

(d) (p. 239) and belongs to the set $\nabla_{2}$, then it also satisfies condition (e).

LEMma 11. We have $P_{0}\left(\nabla_{2}\right)=1$.

Proof. In order to prove this it suffices to show, in view of (103), that for every $T \geqslant 0$

$$
P_{0}\left(\bigcup_{n=0}^{\infty} B_{n}(T)\right)=1
$$

$i$. e., the sets $B_{n}(T)$ being disjoint for different indices $n$, that

$$
\sum_{n=0}^{\infty} P_{0}\left(B_{n}(T)\right)=1
$$

The function $w$ satisfying conditions (a), (b), (c), and (d), $i$. e., belonging to the set $V_{1} \cap[W-A]$, is in the set $B_{r}(T)$ if and only if $w_{2}(\langle 1\rangle) \geqslant$ $\geqslant T$. Thus we deduce from formulae $(83)$ and $(95)$ that the sets $B_{0}(T)$ and $\left\{w_{2}(\langle 1\rangle) \geqslant T\right\}$ are almost equal, whence in virtue of (75)

$$
P_{0}\left(B_{0}(T)\right)=\exp \left(-\pi_{1}^{1} T\right) .
$$

Similarly, we may verify that the sets $B_{1}(T)$ and

(106) $\quad \bigcup_{j=2}^{\infty} \bigcap_{i=1}^{j}\left\{w_{2}(\langle 1,\langle i, j\rangle\rangle) \geqslant T, w_{2}(\langle 1\rangle)<T\right\} \cup$

$$
\cup \bigcap_{j=2}^{\infty} \bigcap_{i=1}^{j}\left\{w_{1}(\langle 1,\langle i, j\rangle\rangle)=w_{2}(\langle 1,\langle i, j\rangle\rangle), w_{2}(\langle 1\rangle)<T\right\}
$$

are almost equal. Taking into account, in view of condition (b), the fact that the summands in (106) are almost disjoint we obtain according to formula (77)

$$
\text { (107) } \begin{aligned}
P_{0}\left(B_{1}(T)\right)= & \sum_{j=2}^{\infty} P_{0}\left(\bigcap_{i=1}^{j}\left\{w_{2}(\langle 1,\langle i, j\rangle\rangle) \geqslant T, w_{2}(\langle 1\rangle)<T\right\}\right)+ \\
& +P_{0}\left(\bigcap _ { j = 2 } ^ { \infty } \bigcap _ { i = 1 } ^ { j } \left\{w_{1}(\langle 1,\langle i, j\rangle\rangle)=\right.\right. \\
& \left.\left.=w_{2}(\langle 1,\langle i, j\rangle\rangle), w_{2}(\langle 1\rangle)<T\right\}\right) \\
= & \sum_{j \neq 1}^{\infty} \pi_{1}^{j} \int_{0}^{T} \exp \left(-\pi_{1}^{1} t\right) \exp \left(-j \pi_{1}^{1}(T-t)\right) d t .
\end{aligned}
$$


Suppose now that $n \geqslant 1$. If $w \in V_{1} \cap[W-A]$ and $w \in B_{n+1}(T)$, then $w_{2}(\langle 1\rangle)<T$, whence the sets $B_{n+1}(T)$ and

$$
\begin{aligned}
\bigcup_{\xi_{1}, \ldots, \xi_{n} \neq\langle 1\rangle} \bigcap_{r=1}^{n}\left\{w_{1}\left(\xi_{r}\right)<w_{2}\left(\xi_{r}\right)<T\right\} \cup \\
\qquad \bigcap_{\eta \neq \xi_{1}, \ldots, \xi_{n},\langle 1\rangle}\left\{\left\{w_{1}(\eta) \neq w_{2}(\eta)\right\} \cup\left\{w_{2}(\eta) \geqslant T\right\} \mid\right.
\end{aligned}
$$

are almost identical. Setting

$$
\begin{aligned}
& \text { (109) } \quad B_{k}^{(i, j)}(T)=\bigcup_{\xi_{1}, \ldots, \xi_{k} \in \mathbb{K}(\langle 1,\langle i, j\rangle\rangle)} \bigcap_{r=1}^{k}\left\{w_{1}\left(\xi_{r}\right)<w_{2}\left(\xi_{r}\right)<I^{\prime}\right\} \frown \\
& \cap \bigcap_{\substack{\eta \in K(\langle 1,\langle i, j\rangle\rangle) \\
\eta \neq \xi_{1}, \ldots, \xi_{k}}}\left\{\left\{w_{1}(\eta)=w_{2}(\eta)\right\} \cup\left\{w_{2}(\eta) \geqslant T\right\}\right]
\end{aligned}
$$

we may represent the set (108) as the union

$$
\bigcup_{j=2}^{\infty} \bigcup_{n_{1}+\ldots+n_{j}=n} \bigcap_{i=1}^{j} B_{n_{i}}^{(i, j)}(T),
$$

in which the summands are almost disjoint, conditions (a), (b), (c), and (d) being satisfied almost everywhere. Thus we have

$$
P_{0}\left(B_{n+1}(T)\right)=\sum_{j=2}^{\infty} \sum_{n_{1}+\ldots+n_{j}=n} P_{0}\left(\bigcap_{i=1}^{j} B_{n_{i}}^{(i, j)}(T)\right) .
$$

By definition (109)

$$
\begin{gathered}
B_{k}^{(i, j)}(T) \epsilon \mathfrak{B}(K(\langle 1,\langle i, j\rangle\rangle)), \\
\mathcal{L}_{\langle 1,\langle i, j\rangle\rangle} B_{k}^{(i, j)}(T)=B_{k}(T), \quad \delta\left(\bigcap_{i=1}^{j} B_{n_{i}}^{(i, j)}(t)\right)=0 .
\end{gathered}
$$

On the other hand, it follows from (102) that $\tau_{t} B_{k}(T)=B_{k}(I-t), B_{k}(t)=0$ for $t<0$. Taking into account these formulae we obtain from (82) the probability

$$
P_{0}\left(\bigcap_{i=1}^{j} B_{n_{i}}^{(i, j)}(T)\right)=\pi_{1}^{j} \int_{0}^{T^{\prime}} \exp \left(-\pi_{1}^{1} t\right) \prod_{i=1}^{j} P_{0}\left(B_{n_{i}}\left(T^{\prime}-t\right)\right) d t
$$

Inserting the expression obtained into formula (110) we get for $n \geqslant 1$

$$
\text { (111) } \quad P_{0}\left(B_{n+1}(T)\right)=\sum_{j=2}^{\infty} \pi_{1}^{j} \int_{0}^{T} \exp \left(-\pi_{1}^{1} t\right)\left[\sum_{n_{1}+\ldots+n_{j=n}} \prod_{i=1}^{j} P_{0}\left(B_{n_{i}}\left(L^{\prime}-t\right)\right)\right] d t
$$

We see from (98), (99), (105), and (107) that $P_{0}\left(B_{0}(T)\right)=Q_{1}\left(0, I^{\prime}\right)$, $P_{0}\left(B_{1}(T)\right)=Q_{1}\left(1, T^{\prime}\right)$, whence in view of the recurrence formulae (100) and (111) $P_{0} B_{n}(T)=Q_{1}(n, T)(n=0,1, \ldots)$. Hence by (101) we obtain (104), which proves the lemma.
We see from (83), (94), and (103) that the function $w$ belongs to the set $V_{1} \cap V_{2} \cap[W-A]$ if and only if it satisfies conditions (a), (b), (c), (d), and (e) (section 3 ) and $w_{1}(\xi) \leqslant w_{2}(\xi)$ for all $\xi \epsilon \xi$; thus the pair $\left\langle w_{1}(\xi), w_{2}(\xi)\right\rangle$ may be considered as the interval $\left[w_{1}(\xi), w_{2}(\xi)\right)$. Hence we have $V=V_{1} \cap V_{2} \cap[W-A]$. By formulae (83), (94), and (103) we deduce hence the measurability of the set $V: V \epsilon \mathfrak{B}_{W}$.

It follows from (84), Lemma 11, and the corollary to Lemma 10 that $P_{0}(V)=1$. The relativization of the $\sigma$-measure $P_{0}$ to the set $\nabla$ gives a normed $\sigma$-measure $P$ defined on the $\sigma$-field $\beta_{F}$ (compare section 7 ).

11. We shall now prove that the $\sigma$-measure $P$ generates a conditional probability (in the sense of section 1 ) satisfying conditions (I), (II), and (III) (compare section 4).

Let us set for $A \epsilon\urcorner 3(k(\xi), t)$ and $B \epsilon 93(\xi-K(\xi), 0)$

$$
P(A \mid\{t \in v(\xi)\} \cap B)=P\left(\tau_{t} \mathscr{L}_{\xi} A\right) .
$$

We observe that, $t, \xi, B$ being fixed, the function $P(A \mid\{t \epsilon v(\xi)\} \cap B$ is a normed $\sigma$-measure on $\beta(K(\xi), t)$. The transformations $\tau_{t}$ and $\mathcal{L}_{\xi}$ coincide on the $\sigma$-field $\cap(K(\xi), t)$ with the transformations $T_{t}$ and $L_{\xi}$ respectively. Hence it follows directly from definition (112) that the function $P(A \mid\{t \in v(\xi)\} \cap B)$ satisfies conditions (I), '(II), and (III). In order to prove that it is equal to the conditional probability induced by the probability $P$, it is sufficient to prove that for $A \epsilon \Im(K(\xi), t)$ and $B \epsilon\urcorner(\xi-K(\xi), 0)$ the equality

$$
P(A \cap\{t \epsilon v(\xi)\} \cap B)=P\left(\tau_{t} \mathcal{L}_{\xi} \mathcal{A}\right) P(\{t \epsilon v(\xi)\} \cap B)
$$

is valid.

Suppose that equality (113) is proved for the sets $A \epsilon \ni(K(\xi), t)$ of the form

$$
\bigcap_{i=1}^{m}\left\{v_{1}\left(\xi_{\iota}\right)<a_{i}, v_{2}\left(\xi_{i}\right)<b_{i}\right\},
$$

where $v_{1}(\eta)$ and $v_{2}(\eta)$ denote the left and the right bound of the interval $v(\eta)$ respectively. Then equality (113) is valid for the sets $A$ of the form

$$
\bigcap_{i=1}^{m}\left\{a_{i}^{(1)} \leqslant v\left(\xi_{i}\right)<a_{i}^{(2)}, b_{i}^{(1)} \leqslant v\left(\xi_{i}\right)<b_{i}^{(2)}\right\}
$$

and for finite disjoint unions of sets of this form. It is easily seen that equality (113) is valid for the limit of the monotone sequence $A_{1}, A, \ldots$ of sets if it is valid for each of the sets $A_{i}$. The finite disjoint unions of the sets (115) give the smallest field containing the sets of form (20). Thus we see that the class of the sets $A \epsilon 73(K(\xi), t)$ satisfying (113) is monotone and contains the smallest field spanned upon the sets of 
form $(20)$ contained in $93(K(\xi), t)$, whence it is identical with the $\sigma$-field $73(K(\xi), t)$ (compare the proof of Lemma 8). It follows that it is sufficient to prove that equality (113) is valid for the sets $A$ of form (114). Analogously, we may state that it is sufficient to prove equality (113) for the sets $B$ of form (114). The proof for these sets will be carried out by induction with respect to the length of the element $\xi$.

(a) Suppose first that the length of the element $\xi$ is equal to $1, i . e$., that $\xi=\langle 1\rangle$. Then the $\sigma$-field $79(\xi-K(\xi), 0)=73(\xi-\Xi, 0)$ consists of the empty set and the entire set $V$. If $B$ is equal to the empty set, then (113) is trivially satisfied, for both sides of the formula are equal to zero. Suppose now that $B=V$; then the equality to be proved may be written as

$$
P(A \cap\{t \in v(\langle 1\rangle)\})=P\left(\tau_{t} A\right) P(t \in v(\langle 1\rangle)) .
$$

Every set $A \epsilon\urcorner 3_{V}$ of form (114) may be represented as the intersection $A=A_{0} \cap\left\{v_{2}(\langle 1\rangle)<T\right\}$ where $A_{0} \epsilon 93(\Xi-(\langle 1\rangle), 0)$.

If $T \leqslant t$, then $\left\{v_{2}(\langle 1\rangle)<T\right\} \cap\{t \in v(\langle 1\rangle)\}=0$ and $\tau_{t}\left\{v_{2}(\langle 1\rangle)<T\right\}$ $C\left\{v_{2}(\langle 1\rangle) \leqslant 0\right\}=0$, whence (116) is satisfied, for both sides of the equality are equal to zero. Suppose now that $T>t$. Then

$$
\begin{aligned}
A \cap\{t \in v(\langle 1\rangle)\} & =A_{0} \cap\left\{v_{2}(\langle 1\rangle)<T\right\}-A_{0} \cap\left\{v_{2}(\langle 1\rangle) \leqslant t\right\}, \\
\tau_{t} A & =\tau_{t} A_{0} \cap\left\{v_{2}(\langle 1\rangle)<T-t\right\} .
\end{aligned}
$$

By formula (80) we get hence

$$
\begin{aligned}
P(A \cap\{t \in v(\langle 1\rangle)\}) & =P\left(A_{0} \cap\left\{v_{2}(\langle 1\rangle)<T\right\}\right)-P\left(A_{0} \cap\left\{v_{2}(\langle 1\rangle) \leqslant t\right\}\right) \\
& =P\left(\tau_{t} A_{0}\right) \exp \left(-\pi_{1}^{1} t\right)-P\left(\tau_{T} A_{0}\right) \exp \left(-\pi_{1}^{1} T\right), \\
P\left(\tau_{t} A\right) & =P\left(\tau_{t} A_{0} \cap\left\{v_{2}(\langle 1\rangle)<T-t\right\}\right) \\
& =P\left(\tau_{t} A_{0}\right)-P\left(\tau_{T} A\right) \exp \left(-\pi_{1}^{1}(T-t)\right) .
\end{aligned}
$$

It follows that $P\left(A \cap\{t \in v(\langle 1\rangle)\}=P\left(\tau_{t} A\right) \exp \left(-\pi_{1}^{1} t\right)\right.$, which gives (116) in virtue of (75). Thus equality (113) is proved in the case $\xi=\langle 1\rangle$.

( $\beta$ ) Suppose now that

$$
\xi=\langle 1,\langle i, j\rangle, \ldots\rangle \neq\langle 1\rangle
$$

and that (113) is valid for elements of a length less than that of $\xi$. It follows from notation (118) that $A \epsilon \cap \beta(K(\xi), t)$ implies

$$
\begin{gathered}
A \epsilon\rceil 3(K(\langle 1,\langle i, j\rangle\rangle), 0), \\
\left.\tau_{t} \mathcal{Q}_{\langle 1,\langle i, j\rangle\rangle} A \epsilon\right\urcorner \xi(K(\xi-\langle 1,\langle i, j\rangle\rangle), t-\tau) .
\end{gathered}
$$

Every set $B \epsilon\urcorner \xi(\xi-K(\xi), 0)$ of form (114) may be represented as the intersection

$$
B=\bigcap_{m} \bigcap_{r=1}^{m} B_{r, m} \cap\left\{v_{2}(\langle 1\rangle)\langle T\} \quad \text { where } \quad B_{r, m} \in\right\urcorner 3(K(\langle\mathbf{1},\langle r, m\rangle\rangle), 0) .
$$

If $T \geqslant t$, then $\left\{v_{2}(\langle 1\rangle)<T\right\} \frown\{t \in v(\xi)\}=\{t \in v(\xi)\} \frown\left\{v_{2}(\langle 1\rangle)<t\right\}$. Thus we may suppose in the proof that

$$
T<t .
$$

We get by $(80)$ and $(82)$

$$
\begin{aligned}
& \text { (121) } P(A \cap\{t \in v(\xi)\} \cap B)=P(A \cap\{t \in v(\xi)\}) \cap \bigcap_{m} \bigcap_{r=1}^{m} B_{r, m}- \\
& -P\left(\tau_{T} A \cap\{t-T \epsilon v(\xi)\} \cap \bigcap_{m} \bigcap_{r=1}^{m} \tau_{T} B_{r, m}\right) \exp \left(-\pi_{1}^{1} T\right) \\
& =\pi_{1}^{j} \int_{0}^{l} \exp \left(-\pi_{1}^{1} \tau\right) P\left(\tau_{t} \mathcal{L}_{\langle 1,\langle i, j\rangle} A \cap\{t-\tau \in v(\xi-\langle 1,\langle i, j\rangle\rangle)\} \cap\right. \\
& \left.\frown \tau_{\tau} \mathscr{L}_{\langle 1,\langle i, j\rangle\rangle} B_{i, j}\right) \prod_{s \neq i} P\left(\tau_{\tau} \mathcal{L}_{\langle 1,\langle s, j\rangle} B_{s, j}\right) d \tau+ \\
& +\sum_{m \neq j} \pi_{1}^{m} \int_{0}^{l} \exp \left(-\pi_{1}^{1} \tau\right) \prod_{r=1}^{m} P\left(\tau_{t} \varrho_{\langle 1,\langle r, m\rangle\rangle} B_{r, m}\right) d \tau- \\
& -\exp \left(-\pi_{1}^{1} T\right) \pi_{1}^{j} \int_{0}^{t-T} \exp \left(-\pi_{1}^{1} \tau\right) P\left(\tau_{T_{+\tau}} \mathcal{L}_{\langle 1,\langle i, j\rangle\rangle} A\right) \cap \\
& \left.\frown\{t-\stackrel{0}{T}-\tau \in v(\xi-\langle 1,\langle i, j\rangle\rangle)\} \cap \tau_{T_{+\tau}} \mathcal{Q}_{\langle 1,\langle i, j\rangle\rangle} B_{i, j}\right) d \tau- \\
& -\exp \left(-\pi_{1}^{1} T\right) \sum_{m \neq j} \pi_{1}^{m} \int_{0}^{t-T} \exp \left(-\pi_{1}^{1} \tau\right) \prod_{r=1}^{m} P\left(\tau_{T+\tau} \varrho_{\langle 1,\langle r, m\rangle} B_{r, m}\right) d \tau, \\
& P(\{t \in v(\xi)\} \cap B)=P\left(\{t \in v(\xi)\} \cap \bigcap_{m} \bigcap_{r=1}^{m} B_{r, m}\right)- \\
& -\exp \left(-\pi_{1}^{1} T\right) P\left(\{t-T \epsilon v(\xi)\} \cap \bigcap_{m} \bigcap_{r=1}^{m} \tau_{T} B_{r, m}\right) \\
& =\pi_{1}^{j} \int_{0}^{t} \exp \left(-\pi_{1}^{1} \tau\right) P\left(\{t-\tau \epsilon v(\xi-\langle 1,\langle i, j\rangle)\rangle) \cap \tau_{\tau} \mathcal{L}_{\langle 1,\langle i, j\rangle\rangle} B_{i, j}\right) \times \\
& +\sum_{m \neq j} \pi_{1}^{m} \int_{0}^{l} \exp \left(-\pi_{1}^{1} \tau\right) \prod_{r=1}^{m} P\left(\tau_{t} \mathcal{L}_{\langle 1,\langle r, m\rangle} B_{r, m}\right) d \tau- \\
& \prod_{s \neq i} P\left(\tau_{\tau} \mathcal{L}_{\langle 1,\langle s, j\rangle} B_{s, j}\right) d \tau+ \\
& -\exp \left(-\pi_{1}^{1} T\right) \pi_{1}^{j} \int_{0}^{t-T} \exp \left(-\pi_{1}^{1} \tau\right) P(\{t-T-\tau \epsilon v(\xi-\langle 1,\langle i, j\rangle\rangle)\} \cap \\
& \left.\frown \tau_{T+\tau} \mathcal{L}_{\langle 1,\langle i, j\rangle\rangle} B_{i, j}\right) \cdot \prod_{s \neq i} P\left(\tau_{T+\tau} \mathcal{L}_{\langle 1,\langle s, j\rangle} B_{s, j}\right) d \tau- \\
& -\exp \left(-\pi_{1}^{1} \bar{T}\right) \sum_{m \neq j} \pi_{1}^{m} \int_{0}^{t-T} \exp \left(-\pi_{1}^{1} \tau\right) \prod_{r=1}^{m} P\left(\tau_{T+\tau} \mathcal{L}_{\langle 1,\langle r, m\rangle\rangle} B_{r, m}\right) d \tau .
\end{aligned}
$$


By the hypothesis of induction

$$
\begin{aligned}
& P\left(\tau_{\tau} \dot{\mathcal{L}}_{\langle 1,\langle i, j\rangle\rangle} A \cap\{t-\tau \epsilon v(\xi-\langle 1,\langle i, j\rangle\rangle)\} \cap \tau_{\tau} \mathcal{L}_{\langle 1,\langle i, j\rangle\rangle} B_{i, j}\right) \\
= & P\left(\tau_{t-\tau} \tau_{r} A\right) P\left(\{t-\tau \epsilon v(\xi-\langle 1,\langle i, j\rangle\rangle)\} \cap \tau_{\tau} \mathcal{L}_{\langle 1,\langle i, j\rangle\rangle} B_{i, j},\right.
\end{aligned}
$$

for the length of the element $\xi-\langle 1,\langle i, j\rangle\rangle$ is less than the length of $\xi$ and (119) is satisfied. For $\tau<t$ the equality $\tau_{t-\tau} \tau_{\tau} A=\tau_{t} A$ is satisfied. Making use of this equality in formula (123), inserting the expression obtained into (121), and extracting the common factor $P\left(\tau_{l} A\right)$, we obtain as the second factor the expression on the right side of formula (122). This proves equality (113), which concludes the proof of the Lemma.

It follows from (113) that the function defined by (112) is equal to the conditional probability induced by the probability $P$ and satisfying conditions (I), (II), and (III). Thus the system $\left\langle V, C_{V}, P\right\rangle$ is a cascade process.

12. We shall now prove that the intensities of the process $\left\langle\Omega(V), 7_{Q(V)}, \Omega P\right\rangle$ (which by Theorem 1 is a homogeneous regular Markov process with a denumerable multitude of states, satisfying conditions (13) and (14)) are equal to $\pi_{1}^{0}, \pi_{1}^{1}, \pi_{1}^{2}, \ldots$

By definition (21) of the function $\omega(v, t)$ it follows that

$$
\begin{aligned}
& E_{0}=\bigcap_{j=2} \bigcap_{i=1}^{j}\left\{v_{1}(\langle 1,\langle i, j\rangle\rangle)=v_{2}(\langle 1,\langle i, j\rangle\rangle), v_{2}(\langle 1\rangle)\right.\leqslant T\} こ \\
& \subset\{\omega(v, T)=0\},
\end{aligned}
$$

(125) $\quad E_{1}=\left\{v_{2}(\langle 1\rangle)>T\{\subset\{\omega(v, T)=1\}\right.$,

$$
\begin{aligned}
E_{k}=\bigcap_{i=1}^{k}\left\{v_{2}(\langle 1,\langle i, k\rangle\rangle)>T\right. & \left., v_{1}(\langle 1,\langle i, k\rangle\rangle) \leqslant T\right\} \subset \\
& \quad\{\{\omega(v, T)=k\} \quad \text { for } \quad k=2,3, \ldots
\end{aligned}
$$

From formulae (75), (80), and (82) we get the probabilities

$$
\begin{aligned}
& P\left(E_{0}\right)=\pi_{1}^{0} / \pi_{1}^{1}\left(1-\exp \left(-\pi_{1}^{1} T\right)\right), \quad P\left(E_{1}\right)=\exp \left(-\pi_{1}^{1} T\right), \\
& P\left(E_{k}\right)=\pi_{1}^{k} \int_{0}^{T} \exp \left(-\pi_{1}^{1} t\right) \exp \left(-k \pi_{1}^{1}(T-t)\right) d t \quad(k \geqslant 2),
\end{aligned}
$$

whence, in view of inclusions (124), (1.25), and (126),

$$
\begin{gathered}
a_{1}^{k}=\lim _{T \rightarrow 0+} \frac{P(\omega(v, T)=k)}{T} \geqslant \pi_{1}^{k} \quad \text { for } k \neq 1 . \\
a_{1}^{1}=\lim _{T \rightarrow 0+} \frac{1-P(\omega(v, T)=\dot{1})}{T} \leqslant \pi_{1}^{1} .
\end{gathered}
$$

Thus we have the inequality

$$
a_{1}^{1}=\sum_{k \neq 1} a_{1}^{k} \geqslant \sum_{k \neq 1} \pi_{1}^{k}=\pi_{1}^{1},
$$

which, together with (127), implies $a_{1}^{1}=\pi_{1}^{1}$. It follows from this equality and the foregoing inequalities that $a_{1}^{k}=\pi_{1}^{k}(k=0,1, \ldots)$.

Thus we have proved that intensities of the process $\left\langle\Omega(V), \mathrm{B}_{\Omega(V)}, \Omega P\right\rangle$ are equal to $\pi_{1}^{0}, \pi_{1}^{1}, \pi_{1}^{2}, \ldots$ The remark in section 7 implies Theorem 2 .

\section{References}

[1] Н.' А. Д митрев и А. Н. Колмогоров, Ветвяииеся случайные прочессьи, Докл. Акад. Наук ССCP 5 (1) (1947), p. 7 - 10.

[2] J. L. Doob, Markoff ehains - denumerable case. Trans. Amer. Math. Soc. 58 (1945), p. $455 \cdot 473$.

[3] - Stochastic processes, New York-London 1953.

[4] - Topics in the theory of Markoff chains, Trans. Amer. Math. Soc. 52 (1952), p. $37-64$.

[5] W. Feller, On the theory of stochastic processes, with particular reference to applications, Proc. Berkeley Symp. Math. Statistics and Probability 1949, p. 403.432.

[6] P. R. Halmos, Measure theory, New York 1950.

[7] А. Н. Колм огоров, $K$ вопросу о дифференцируемости переходних веролтностей в однородных по времени прочессах Маркова со сиетным числом со. столний, Ученые Записки Моск. Унив., вып. 148 (1951), p. 53.59.

[8] E. Marczewski, On compact measures, Fund. Math. 40 (1953), p. 113.124.

[9] K. Urbanik, On a stochastic model of a cascade, Bull. Acad. Pol. Sei., cl. III, 3.7 (1955), p. $349 \cdot 351$

INSTYTUT MATEMATYCZNY POLSKIEJ AKADEMII NAUK

MATHEMATICAL INSTITUTE OF THE POLISH ACADEMY OF SCIENCES

Reçu par la Rédaction le 23. 7. 1956 